\title{
The combined action of CTCF and its testis-specific paralog BORIS is essential for spermatogenesis
}

Samuel Rivero-Hinojosa ${ }^{1,2^{*}}$, Elena M. Pugacheva ${ }^{1^{*}}$, Sungyun Kang ${ }^{1,3}$, Claudia Fabiola MéndezCatalá $^{1,4}$, Alexander L. Kovalchuk ${ }^{1}$, Alexander V. Strunnikov ${ }^{5}$, Dmitri Loukinov ${ }^{1}$, Jeannie T. Lee ${ }^{6}$, Victor V. Lobanenkov ${ }^{*}$

${ }^{1}$ Laboratory of Immunogenetics, National Institute of Allergy and Infectious Diseases, National Institutes of Health, Bethesda, MD 20892, USA.

${ }^{2}$ Center for Cancer and Immunology Research, Children's National Research Institute, Washington, DC 20010, USA

${ }^{3}$ Department of Biology, Indiana University, Bloomington, IN 47405, USA

${ }^{4}$ Laboratory of Genetics and Molecular Oncology, Building A4, Faculty of Higher Studies (FES) Iztacala, National Autonomous University of Mexico (UNAM), Tlalnepantla, State of Mexico, Mexico.

${ }^{5}$ Guangzhou Institutes of Biomedicine and Health, Molecular Epigenetics Laboratory, 190 Kai Yuan Avenue, Science Park, Guangzhou 510530, China;

${ }^{6}$ Department of Molecular Biology, Massachusetts General Hospital; Department of Genetics, Harvard Medical School, Boston, MA 02114, United States of America;

*Lead Contact. To whom correspondence may be addressed: Samuel Rivero-Hinojosa (sriverohin@childrensnational.org), Elena M. Pugacheva (epugacheva@niaid.nih.gov), Victor V. Lobanenkov (vlobanenkov@niaid.nih.gov) 


\begin{abstract}
CTCF is a key organizer of the 3D genome. Its specialized paralog, BORIS, heterodimerizes with CTCF but is expressed only in male germ cells and in cancer states. Unexpectedly, BORIS-null mice have only minimal germ cell defects. To understand the CTCF-BORIS relationship, mouse models with varied CTCF and BORIS levels were generated. Whereas $\mathrm{Ctcf}^{++} \mathrm{Boris}^{+/+}$, $\mathrm{Ctcf}^{+/-}$ Boris $^{+/}$, and $\mathrm{Ctcf}^{+/+}$Boris $^{-/}$males are fertile, Ctcf ${ }^{+-}$Boris $^{-/-}$(Compound Mutant; CM) males are sterile. Testes with combined depletion of both CTCF and BORIS show reduced size, defective meiotic recombination, increased apoptosis, and malformed spermatozoa. Although CM germ cells exhibit only $25 \%$ of CTCF WT expression, chromatin binding of CTCF is preferentially lost from CTCF-BORIS heterodimeric sites. Furthermore, CM testes lose the expression of a large number of spermatogenesis genes and gain the expression of developmentally inappropriate genes that are "toxic" to fertility. Thus, a combined action of CTCF and BORIS is required to both repress pre-meiotic genes and activate post-meiotic genes for a complete spermatogenesis program.
\end{abstract}




\section{INTRODUCTION}

How the 3D genome is organized through diverse developmental stages is one of the key questions in the epigenetics field. The CCCTC-binding factor (CTCF) is a chief organizer of chromosome looping in multicellular eukaryotes ranging from insects to humans ${ }^{1,2}$. CTCF is an exceptionally conserved 11-Zinc Finger (ZFs) DNA-binding protein that binds thousands of genomic sites in the fly, mouse, and human genomes ${ }^{3-5}$. CTCF functions by halting cohesinmediated extrusion of chromatin fibers thus creating chromosome loops ${ }^{6-8}$. CTCF thereby partitions the genome into autonomously functioning expression units ${ }^{2,9}$. Numerous nuclear processes including transcription regulation, imprinting, chromatin insulation, X-chromosome inactivation, and repair of DNA double-strand breaks (DSBs) have been linked to the organizational function of CTCF ${ }^{10-21}$. The essential function of CTCF is demonstrated by the early embryonic lethality of mice that are null for $\mathrm{Ctcf}^{2}$. Furthermore, although heterozygous Ctcf knockout $\left(\mathrm{Ctcf}^{+-}\right)$mice are born at the expected Mendelian ratio and show no obvious developmental defects $22-24$, the haploinsufficiency results in a predisposition to spontaneous tumorigenesis in a broad range of tissues ${ }^{25}$. Proper expression and function of CTCF are therefore essential both during development and in the adult soma.

The Ctcf gene has a close paralog known as Boris (Brother Of the Regulator of Imprinted Sites) or Ctcfl (CCCTC-binding Factor-Like) ${ }^{26,27}$ that appeared later in evolution as a duplication of Ctcf in early vertebrates ${ }^{28}$. The two genes share a high degree of homology in the central 11Zinc finger DNA binding domain. As a consequence, CTCF and BORIS proteins bind to virtually the same DNA motifs in vivo and in vitro ${ }^{29-33}$, however, they differ dramatically in their $\mathrm{N}$ - and Cterminal domains ${ }^{34}$. Recent studies show that CTCF and BORIS interact with different protein partners ${ }^{35-37}$. Notably, the divergence of the $\mathrm{N}$-terminal region of the two proteins prevents BORIS from being able to block cohesin-mediated loop extrusion in contrast to CTCF ${ }^{36,37}$.

Altogether, these observations suggest different developmental functions for the two paralogs. Indeed, whereas CTCF expression is ubiquitous, BORIS expression is normally restricted to male germ cells ${ }^{27}$. In mice, the testis is the only tissue affected to some extent by a Boris knockout ${ }^{32}$. Nevertheless, Boris knockout male mice have only minor spermatogenesis defects, including a reduction of testicular size due to increased apoptosis in germ cells and delayed production of haploid cells ${ }^{29,31,32}$. Surprisingly, Boris-null males are still fertile, though they exhibit sub-fertility. 
Therefore, it is unclear at present what is the BORIS's exact molecular and developmental role and whether its close relationship to CTCF may account for the partial penetrance of Boris $\mathrm{KO}$ in male germline. These questions are significant not only in a developmental context but also for the development of cancer. It is now well established that Ctcf haploinsufficiency predisposes to cancer ${ }^{25}$. It is also known that BORIS is aberrantly activated in many types of cancer ${ }^{30,35,38-42}$. As such, BORIS has been recognized as a "cancer-testis antigen", but it is unclear whether it has a universal mechanism of action in cancer cells.

With respect to molecular interaction between the two factors, when CTCF and BORIS are co-expressed, either in germline or cancer cells, they bind at a subset of binding regions that contain at least two closely-spaced CTCF binding motifs (termed clustered CTCF Target Sites or $2 x C T S e s)^{33,43}$. In contrast to 1 xCTSes bound only by CTCF, the 2xCTSes predispose the physical and heterodimeric interactions between CTCF and BORIS due to DNA-dependent constrains ${ }^{33,44}$. Interestingly, these heterodimeric sites of binding are enriched at active promoters and enhancers in both germ cells and cancer cells ${ }^{33}$. Moreover, the putative heterodimerization of CTCF and BORIS at the $2 \times C T S e s$ have been shown to be involved in transcriptional program of cancer cells and activation of testes-specific genes in cancer ${ }^{33,43,45}$. In mature spermatozoa, the $2 \times C T S e s$ are associated with retained histones in mice and humans ${ }^{33,46}$. The exact degree of functional integration of CTCF-BORIS heterodimers currently remains unknown, as a systematic genetic analysis has yet to be performed in a normal developmental process - i.e., outside of cancer states.

To advance the understanding of both molecular and functional interaction between CTCF and BORIS, here we describe a genetic model in the male germline of the mouse - the only known normal context in which BORIS is expressed. Mouse spermatogenesis is a unique multistage process that occurs over a 2-week period, during which diploid spermatogonia are transformed into haploid germ cells. The dramatic transformation occurs morphologically, genetically, and transcriptionally, and therefore makes an excellent dynamic model in which to study CTCF-BORIS interactions. In this work, we generated mice with varying levels of CTCF and BORIS and investigated the functional consequences and effects on the spermatogenesis gene expression program. Our findings demonstrate that the CTCF-BORIS interaction is a developmental activator that is essential for induction of male gametogenesis genes. 


\section{RESULTS}

\section{$\mathrm{Ctcf}^{+/-} \mathrm{Boris}^{-/}$male mice are infertile with spermatogenic defects and spermatozoa} abnormalities.

Testis is the only mammalian tissue in which the two paralogous genes, Ctcf and Boris, are normally co-expressed ${ }^{27,41}$. To examine the interplay between CTCF and BORIS during all stages of spermatogenesis, we generated mouse models in which the expression levels of CTCF and BORIS varied in testis. Because a complete Ctcf-deficiency results in embryonic lethality, we were only able to produce mice with heterozygous Ctcf knockout (CTCF HET; Ctcf $^{+-}$). In contrast, complete Boris knockouts (BORIS KO; Boris ${ }^{-/}$) were viable. Thus, four mouse models were created for subsequent analysis: Wild-type (WT; Ctcf $^{++}$Boris $\left.^{+/+}\right)$; CTCF HET $\left(\right.$Ctcf $^{+/-}$Boris $\left.^{+/+}\right)$; BORIS KO ( Ctcf $^{++}$Boris $\left.^{-/}\right)$; and Compound Mutant (CM; Ctcf ${ }^{+-}$Boris $\left.^{-/}\right)$. Animals of each genotype were viable and indistinguishable from their WT littermates in survival rate and appearance. Breeding tests showed that mating behavior of all mouse models was within the norm, and the same frequency of copulation plugs was observed in all genotypes. However, only CM male mice displayed complete infertility (Table 1). Repeated breeding attempts to cross CM males with WT females yielded no offspring, with the exception of one stillborn deformed, five-legged, male pup (Supplementary Figure 1a) that was twice the size of a normal newborn pup. A comparative genomic hybridization at the resolution of $6.5 \mathrm{~kb}$ revealed the presence of $Y$-chromosome material and no notable genomic abnormalities besides Boris heterozygosity in this pup.

Analysis of cauda epididymis showed greatly reduced sperm count in CM male mice (Fig. 1a, Supplementary Figure 1b). In addition to reduced numbers, the CM sperm also manifested poor motility and progressive movement as compared to WT, CTCF HET and BORIS KO mice (Fig.1a). The vast majority of the CM spermatozoa were also morphologically abnormal, with approximately $90-95 \%$ of $\mathrm{CM}$ sperm having tails bent $180^{\circ}$ through the cytoplasmic droplet and $3-5 \%$ having abnormal heads with a round shape (Fig.1b, S Supplementary Figure 1c).

The examination of adult testes showed that testes from 3-month-old CM animals were significantly smaller than those of age-matched WT, CTCF HET, and BORIS KO mice ( $p$ value=2.825e-07) (Fig.1c). Histological analysis revealed that $\mathrm{CM}$ mice had reduced testicular cellularity compared to WT, CTCF HET, and BORIS KO (Fig.1d). In contrast to WT, we observed a relatively large number of empty tubules in $\mathrm{CM}$ testes with reduced numbers of meiotic and post meiotic cells, although some normal tubules were also present in CM testis, indicating certain heterogeneity between tubules and animals (Fig.1d and S Supplementary Figure 1d). Moreover, some of the CM tubules lacked elongated spermatids, suggesting a meiotic arrest at the 
spermatocyte stage. Furthermore, CM testes had numerous multinucleated giant cells (Fig.1e, Supplementary Figure 1d), a feature found in a number of spermatogenesis-defective mouse genotypes ${ }^{47,48}$. Similar multinucleated giant cells were also present in BORIS KO, CTCF HET and WT testis, but in much lower numbers (Fig.1e). A test for apoptosis by Terminal deoxynucleotidyl transferase dUTP Nick End Labeling (TUNEL) staining showed a dramatic increase in the average number of TUNEL-positive cells per seminiferous tubules in CM compared to WT, CTCF HET and BORIS KO mice (Fig.1f). Apoptosis appeared to occur at all steps of spermatogenesis in CM testes (Fig.1f). The increased apoptosis in the testes of CM mice likely accounts for the reduced cellularity and size of the testis. Altogether, these data argue that the sterility of CM mice could be attributed to the combination of high apoptotic rates resulting in low sperm count, and the reduced motility and abnormal morphology of sperm itself.

\section{Ctcf +/-Boris-/- male mice show defects in meiotic recombination.}

Meiosis is the key genetic process in the germline, as during meiosis I, genetic diversity is generated by recombination between paired homologous chromosomes. Pairing of homologs is mediated by the Synaptonemal Complex (SC), a multiprotein meiosis-specific structure necessary for both synapsis and the completion of recombination during meiosis ${ }^{49}$. The increased apoptosis in spermatocytes, the absence of post meiotic cells in some CM tubules, and the presence of multinucleated giant cells in CM seminiferous tubules (Fig.1d, e, f) suggested an aberration at the early steps of spermatogenesis, such as during meiosis I. Surface spreads of spermatocytes isolated from 3 month-old WT and CM testes stained with antibodies specific for lateral (SYCP3) and central (SYCP1) SC components revealed obvious abnormalities in over $40 \%$ of CM spermatocytes, not observed in spermatocytes from WT mice. Particularly, in WT pachytene spermatocytes, all autosomes were fully synapsed, shown by the contiguous and overlapping SYCP3 and SYCP1 signals along the entire length at pachynema (Fig.2a, Supplementary Figure 2a). In CM spermatocytes, although SYCP3 and SYCP1 signals were generally overlapping, SYCP3 also formed abnormal aggregates at some paired chromosomes (Fig.2a, Supplementary Figure $2 \mathrm{a}$, shown by red arrows). These SYCP3 aggregates were observed either at the end or in the middle of paired chromosomes (Fig.2a, b, Supplementary Figure 2) and ranged from 0 to 6 per CM spermatocyte (Fig.2a, b, Supplementary Figure 2). At the same time, $60 \%$ of CM spermatocytes showed apparently normal SC formation (Supplementary Figure 2b), indicating that the SYCP3 aggregation phenotype is either not fully penetrant or represents an accumulation of a transitional stage, not captured in WT mice. 
The second independent molecular hallmark of meiosis I is programmed DNA double strand breaks (DSBs) introduced by endonuclease Spo11 at PRDM9 binding sites in leptonema ${ }^{50}$. DSBs are marked by a phosphorylated form of the variant $\mathrm{H} 2 \mathrm{AX}$ histone $(\gamma-\mathrm{H} 2 \mathrm{AX})^{51}$. Indeed, both WT and CM spermatocytes showed accumulation of $\gamma$-H2AX signal in leptonema and zygonema as a part of normal progression of meiosis I (Fig.2c). During late pachynema and diplonema, DSBs are repaired and consequently $\gamma-\mathrm{H} 2 \mathrm{AX}$ remains only at unsynapsed sex chromosomes forming a meiotic sex vesicle ${ }^{52}$. Accordingly, we detected a specific accumulation of $\gamma-\mathrm{H} 2 \mathrm{AX}$ staining only at the sex vesicle in WT pachytene spermatocytes (Fig.2b, 2c). However, in CM pachytene spermatocytes, we observed an expansion of $\gamma-\mathrm{H} 2 \mathrm{AX}$ signal beyond sex chromosomes, into autosomes (Fig.2b,c, yellow arrows). The accumulation of $\gamma$-H2AX staining on autosomes of CM pachytene spermatocytes may indicate the persistence of unrepaired DSBs beyond early pachynema, as previously demonstrated for Parp 1 knockout ${ }^{53}$ and for DNA repair factor Hus1 inactivation ${ }^{54}$. Nevertheless, the CM spermatocytes that reached diplonema showed normal $\gamma$-H2AX and SYCP3 staining similar to WT (Fig.2c), suggesting that the pachynema spermatocytes with abnormal staining are preferentially eliminated from meiosis, possibly by apoptosis, or recover after a delay.

Taken together, our data indicate that the simultaneous depletion of both CTCF and BORIS in $\mathrm{Ctcf}^{+/}$Boris $^{-/-}$male mice results in some meiotic defects, including abnormal formation of the SC and persistence of unrepaired DNA damage - further providing an underlying basis for the sterility phenotype of CM males.

\section{CTCF and BORIS co-bind at active promoters and enhancers in male germ cells.}

We next assessed the underlying molecular mechanisms of simultaneous CTCF and BORIS depletion in male germ cells. It is logical to assume that the possibility of functional integration of two proteins is the highest in cell types, where their expression is comparable, as has been shown in cancer cell lines ${ }^{33}$. In testis, it is established that CTCF expression spans all stages of spermatogenesis, but reports of BORIS expression in mice and humans have been somewhat conflicting ${ }^{31-33}$. In situ hybridization of human testes showed that BORIS is expressed in multiple isoforms in different stages of spermatogenesis from spermatogonia to round spermatids $^{30}$. Single-cell transcriptomes of human testicular cells from puberty to adults ${ }^{55}$ also demonstrate that CTCF and BORIS are co-expressed in most stages of spermatogenesis, with the highest levels of co-expression in spermatogonia and early spermatocytes (Supplementary Figure 3a). To reconcile the diverse data we reanalyzed single-cell RNA-Seq data of germ cells isolated from mouse testes ${ }^{56}$ and also verified that Ctcf and Boris are co-expressed from early to 
the late stages of spermatogenesis in mice, as well as in humans. Boris expression was highest in undifferentiated spermatogonia, decreased in spermatocytes, and upregulated again in postmeiotic spermatids (Fig.3a).

Earlier studies had reported CTCF and BORIS binding sites in round spermatids and cancer cells ${ }^{33}$. There, CTCF and BORIS were found to co-bind and form heterodimers at subset of genomic regions consisting of at least two closely spaced CTCF binding sites (named $2 x$ CTSes) ${ }^{33}$. Because we were interested in mapping binding sites across multiple developmental stages, including when BORIS levels are highest, we performed ChIP-seq in the whole testes of 3-month-old WT mice. Of note, spermatogonia have the greatest BORIS expression, but they are the rarest cells in testis and thus we could not perform ChIP-seq on this population of germ cells.

In an agreement with previous studies ${ }^{33}$, ChIP-seq showed a selective binding pattern for CTCF (34625 bound regions) and BORIS (7654 bound regions) (Fig.3b,c). Only a minority of CTCF-bound CTCF Target Sites (CTSs) (6257 of 34625 or 18\%) were co-occupied by BORIS (CTCF/BORIS sites), while the majority (28368 of 34625 or $82 \%$ ) of CTSs were bound by CTCF alone (CTCF-only sites). Additionally, we mapped a small number of regions (1397) occupied by BORIS alone (BORIS-only sites). To analyze whether the regions bound by both CTCF and BORIS in mouse testis belong to the class of $2 \times C T S$ binding sites, we carried out CTCF/BORIS motif analysis on the sequences around the summit of either CTCF/BORIS or CTCF-only and BORIS-only ChIP-seq peaks. Indeed, in contrast to $14 \%$ of CTCF-only binding sites, almost $70 \%$ of CTCF/BORIS binding regions enclose at least two CTCF/BORIS binding motifs that allow cobinding and heterodimerization of the two proteins with similar 11-ZF DNA binding domain (Fig.S4) as previously demonstrated ${ }^{33}$. Pathway analysis showed that a number of cellular processes are associated with the genes containing the three types of CTCF and/or BORIS binding sites (Supplementary Data 1).

In agreement with previous reports ${ }^{33,57}$, CTCF/BORIS and BORIS-only binding regions were enriched in promoters, whereas CTCF-only regions were more enriched at intergenic and intragenic genomic regions (Fig.3d). Furthermore, overlapping mouse testis ENCODE epigenomic data with our ChIP-seq data showed that BORIS binding (CTCF/BORIS and BORISonly) was associated with active promoters enriched for the transcription initiating form of RNA polymerase II (RNAPII) and active histone modifications, H3K4me3 and H3K27ac, that mark active promoters and enhancers, respectively. These associations were at regions that are transcriptionally active during spermatogenesis (Supplementary Figure 3b). To the contrary, CTCF-only regions were depleted of RNAPII and active histone marks in mouse testis (Fig.3e). 
Thus, these data indicate an interaction of the two paralogous proteins preferentially at active promoters and enhancers in the male germ cells.

\section{BORIS is required for full CTCF expression in male germ cells.}

The complete sterility of CM, but not CTCF HET or BORIS KO male mice, suggests that normal spermatogenesis proceeds with reduced CTCF levels in the presence of BORIS but is dysregulated when CTCF levels are reduced in the complete absence of BORIS. Incidentally, analysis of ChIP-seq data of CTCF and BORIS genomic occupancy in mouse testis showed that CTCF and BORIS bind their own promoters as well as each other's promoters (Fig.4a), suggesting a possibility that CTCF and BORIS both autoregulate their own transcription and reciprocally regulate each other's transcription. We therefore analyzed Ctcf and Boris expression in testes of 3-month-old mice of the four genotypes by qRT-PCR (Fig.4B). As expected, Ctcf expression was reduced to $50 \%$ in CTCF HET testis compared to WT, where only one Ctcf allele is present (Fig.4b). Surprisingly, Ctcf expression was significantly downregulated to approximately $70 \%$ of the WT expression in BORIS KO testis in which both Ctcf alleles are present (Fig.4b), suggesting that BORIS is necessary for the normal expression of Ctcf. Furthermore, the heterozygosity for Ctcf combined with Boris knockout in $\mathrm{CM}$ testis resulted in a further downregulation of Ctcf expression to only 25\% of WT levels (Fig.4b). Western blotting of CTCF protein levels in testis and spleen from mice of the four genotypes corroborated the mRNA data (Fig.4c). On the other hand, the deletion of the Boris gene had no effect on Ctcf expression in somatic tissues (Supplementary Figure 5). Thus, the requirement for BORIS for full CTCF expression was observed only in testis, and the severity of CM phenotype may well be attributed to the combined effect of haploinsufficiency of Ctcf gene and the absence of BORIS-mediated upregulation of the remaining Ctcf allele.

\section{Preferential loss of CTCF from CTCF/BORIS dimeric sites in CM testes}

Given that CTCF expression decreased dramatically upon Boris knockout, we performed CTCF ChIP-seq to learn how CTCF binding to DNA changes in CM testes, where CTCF levels fell to $25 \%$ of normal. First, to determine if the occupancy of CTCF binding sites is maintained across different types of germ cells in mouse testis, we compared CTCF ChIP-seq in round spermatids and spermatocytes purified from 3-month-old mice with CTCF ChIP-seq from the whole testis. We found that the occupied CTCF binding sites in the whole testis are conserved in the individual types of germ cells: round spermatids and spermatocytes (Supplementary Figure 6). This is not surprising, as CTCF occupancy is generally conserved across different cell types, with only $\sim 20 \%$ of binding sites being cell type specific ${ }^{58}$. Thus, we proceeded with the mapping 
of CTCF binding sites in germ cells isolated from the whole testes of WT and CM mice. In contrast to 34625 CTCF ChIP-seq peaks identified in WT testis (Fig.3b, 5a), only 21525 CTCF peaks were identified in CM testis (Fig.5a, Supplementary Figure 7a,b,c). At 19,000 CTCF sites that were shared between CM and WT testes (Fig.5a), CTCF peaks were 20\% smaller in CM compared to WT (Fig.5b). On the other hand, at 1922 sites the CTCF occupancy is actually increased in CM compared to WT testes (Fig.5a, Supplementary Figure 7a-c), which could be explained by either a slower CTCF turnover at these sites to compensate for the lower amount of available CTCF or by a necessity to maintain a high occupancy at these CTCF sites. Interestingly, we have not observed any significant loss of CTCF occupancy in BORIS KO germ cells, suggesting that the level of CTCF in BORIS KO is functionally sufficient to keep CTCF binding pattern close to that in wild type mice (Supplementary Figure 6d).

As the male sterility phenotype emerged only in CM mice, we also asked whether the loss of CTCF occupancy was specifically associated with CTCF/BORIS sites. K-means ranked clustering of CTCF and BORIS occupancy along 15198 lost CTCF binding sites showed a large cluster of CTCF/BORIS sites that lost CTCF occupancy in CM compared to WT testes (Supplementary Figure 7d). This, in turn, prompted us to analyze CTCF occupancy at CTCF-only and CTCF/BORIS sites in WT and CM testes (Fig.3b). The average plot analysis of normalized tag density showed $25 \%$ reduction of CTCF occupancy at CTCF-only sites ( $p$-value: 7.321477 27, Wilcoxon test) and a much more severe loss (50\%) at CTCF/BORIS sites (p-value: 2.175676e-66, Wilcoxon test) (Fig.5c). Additionally, some CTCF/BORIS sites lost CTCF occupancy completely in CM (Fig.5d). Thus, CTCF binding is preferentially lost from CTCFBORIS dimeric sites which are associated with active transcription in the male germ cells.

To further assess the specificity of CTCF binding loss in CM testis, we combined CTCF peaks mapped in both WT and CM testes into a composite set of CTCF binding sites (36547) and separated them into three groups based on the differential CTCF ChIP-seq tag density as follows: WT>CM sites with the tag density more than two-fold higher in WT than in CM (13385 CTCF peaks), WT<CM sites with greater than two-fold lower CTCF occupancy in WT than in CM (2819 CTCF peaks), and CTCF=CM sites where tag density did not change more than two-fold (7051 CTCF peaks) (Supplementary Figure 7e,f). An analysis of BORIS occupancy in WT testes at CTCF binding regions with differential WT/CM CTCF occupancy demonstrates that the loss of CTCF binding in CM is preferentially associated with CTCF/BORIS binding regions, as WT>CM sites were at least two-fold enriched with BORIS occupancy compared to WT=CM sites (Supplementary Figure $7 \mathrm{~g}$ ). These results also suggest that binding at CTCF/BORIS 
heterodimeric sites has greater stability than CTCF binding alone. An analysis of genomic distribution of differentially bound CTCF regions indicated that the CTCF binding in CM was preferentially lost (WT > CM) at the promoter regions compared to CTCF binding sites that remained bound in CM cells (WT<CM and WT=CM) (Supplementary Figure 7h). Moreover, CTCF binding sites that lost CTCF occupancy in $\mathrm{CM}(\mathrm{WT}>\mathrm{CM})$ were highly associated with transcription regulatory regions as compared to $\mathrm{WT}<\mathrm{CM}$ or $\mathrm{WT}=\mathrm{CM}$ regions, as indicated by the higher enrichment of ChIP-seq tag density of the active transcriptional marks, RNAPII, H3K4me3, and H3K27Ac, specifically at WT>CM regions (Fig.5e). Thus, the lost CTCF binding sites in CM testis are predominately associated with BORIS binding and active transcription marks in WT testes.

\section{Loss of spermatogenesis-specific gene expression in CM germ cells.}

Considering the severe spermatogenesis defects in CM germ cells (Fig.1,2), we used RNA sequencing (RNA-seq) to analyze underlying gene expression changes in testes of our four mouse models at 3 months of age. Significant changes were indeed observed in CM testis. CTCF HET and BORIS KO showed only 0 and 58 differentially expressed genes (DEGs), respectfully (Fig.6a,b, Fold change $>2$, adjusted p-value $<0.001$, Supplementary Data 2), consistent with minimal effects on fertility in these animals. By contrast, CM testis showed 1513 DEGs relative to WT (Fig.6a,b, Supplementary Data 2). RNA-seq results were validated by quantitative RT-PCR on a subset of DEGs (Supplementary Figure 8). DEGs could either be up- or down-regulated, as it is usually observed in such transcriptome-wide analyses, consistent with their being both direct and indirect targets. However, more than 400 genes were downregulated by the loss of Boris and haploinsufficiency of Ctcf, encompassing of $26 \%$ of all DEGs in CM testis.

It is evident, however, that differential gene expression detected in a tissue as a whole could be affected by changing cell type composition. Incidentally, the whole testis contains multiple populations of germ cells that diverge in their stages of growth and differentiation. To ensure that the observed changes in gene expression program were not due to the potential changes of cell-type composition of the CM tubules, we performed RNA-seq analysis of round spermatids and spermatocytes purified from the four mouse genotypes. To validate the enrichment of specific germ cell types used for the RNA-seq analysis, we compared the transcriptional profiles of round spermatids and spermatocytes with a set of previously described markers $^{59}$ and also selected 833 post-meiotic genes from our analysis, which are highly expressed in round spermatids, but not in spermatocytes. Supplementary Figure 9 demonstrates that the expression of round spermatids markers not only distinguishes meiotic (spermatocytes) and post-meiotic (round spermatids) cells in the four mouse genotypes, but also shows that those 
markers are not significantly affected in CM round spermatids compared to other types of mice. Analyzing RNA-seq data from purified round spermatids and spermatocytes of the four mouse genotypes, we confirmed the non-additive impact of Ctcf haploinsufficiency joined with BORIS depletion on the deregulation of multiple genes in CM mice (Fig6b-c). Most importantly, we show that the differentially expressed genes in CM whole testis are deregulated in the same direction in $\mathrm{CM}$ round spermatids and spermatocytes, but to a lesser extend in the latter (Fig.6b). Reciprocally, differentially expressed genes in CM round spermatids are also deregulated in the same direction in the whole testis (Fig.6c), confirming that the RNA-seq analyses of both the individual germ cell types and the whole testis are valid and complementary to each other. Thus, we suggest that the massive transcriptional deregulation observed in whole testis and in the main germ cell types of CM mice is a consequence of the simultaneous depletion of both CTCF and BORIS proteins.

Gene ontology analysis revealed that the downregulated genes in CM whole testis were significantly associated with sexual reproduction, spermatogenesis, male gamete generation, spermatid differentiation, and fertilization (Fig.6d, Supplementary Data 3). By contrast, upregulated genes were not associated with a uniform pathway (e.g., oxidation/reduction, blood vessel development, lipid biosynthesis process, immune response, apoptosis (Fig.6d and Supplementary Data 4). To confirm the effect of combined CTCF and BORIS depletion at pathway level, we performed a Gene Set Enrichment Analysis (GSEA) comparing CTCF HET, BORIS KO and CM to WT testis. The top 10 downregulated pathways related to spermatogenesis in CM testis were also analyzed in CTCF HET and BORIS KO testes. As indicated in Supplementary Figure 10, the normalized enrichment scores (NES) were significantly higher in CM compared to CTCF HET and BORIS KO, confirming the non-additive impact on the pathways associated with spermatogenesis. Thus, there was a general negative effect on the spermatogenesis gene expression program in CM testis, suggesting that BORIS and CTCF must normally cooperate to regulate the spermatogenesis program.

Among the downregulated DEGs in CM are some confirmed direct targets of CTCF and BORIS, such as Cst (Gal3st1), Tsp50 (Prss50) and FerT (Fert2) ${ }^{29,32,60}$ These genes were not only downregulated in the whole testis but also in such individual germ cells types as round spermatids and spermatocytes (Supplementary Figure 11a). The combined effect of simultaneous CTCF and BORIS depletion was even more obvious comparing DEGs in CM to BORIS KO testes, with the majority of DEGs being affected in a similar way but more profoundly in CM compared to BORIS KO testes (Fig.6b). Notably, the most highly down- and up-regulated genes in CM testis 
compared to WT were also down-regulated and up-regulated in BORIS KO testis (Fig.6b, Supplementary Data 2). These data demonstrate that CTCF expression can partially compensate for the loss of BORIS in the BORIS KO, but when CTCF expression is reduced in CM mice, it results in a much stronger impact on the spermatogenesis transcription program. These dramatic changes could therefore explain the infertility of CM males.

To ask if altered CTCF/BORIS occupancy in CM testis could be the basis of differential gene expression, we integrated ChIP-seq and RNA-seq data for WT versus CM. Significantly, 37\% (557 genes out of 1513, Fisher exact test: $p$-value=0.0001) of DEGs in CM testis had CTCF or/and BORIS binding within or in close proximity to the genes $( \pm 2 k b)$ in WT testis (Supplementary Figure 11b, Supplementary Data 5). This association suggested that the loss of CTCF/BORIS binding might underlie the differential expression. Therefore, we examined some previously described CTCF/BORIS target genes. For example, Prss50 harbors a CTCF and BORIS heterodimeric site (2xCTS) in the promoter region ${ }^{29}$. In BORIS KO testis, we observed a $\sim 1.5$-fold downregulation of Prss50 expression; by contrast, we found $>5$-fold downregulation of Prss50 expression in CM testis versus WT testis (Fig.6e, f, Supplementary Figure 11a). Similar patterns were observed for other genes including Mfi2, with a severe downregulation of expression in CM testis (Fig.6e, f and Supplementary Figure 12a,b).

Overall, among all DEGs (1513), 475 (32\%) genes were bound by CTCF in the promoter region (TSS+2kb) in WT cells, and almost half of these $(69 \%, 327$ genes, Fisher exact test, $p$ value $=0.0048$ ) showed a loss of CTCF binding in CM cells (Supplementary Data 5), pointing to a direct impact of CTCF binding on gene transcription in testis. Similarly, among 605 DEGs found in the round spermatids of CM testis, 205 (34\%) genes were bound by CTCF in the promoter region $(\mathrm{TSS}+2 \mathrm{~kb})$ in WT cells, and 157 of them $(77 \%$, Fisher exact test, $\mathrm{p}$-value=0.071) showed a loss of CTCF binding in CM cells (Fig.6c). To further refine the GO analysis of DEGs, we sorted the genes based on CTCF and BORIS occupancy and with the respect to the loss of CTCF occupancy in CM testis. As shown in Supplementary Figure 13a, the spermatogenesis related pathways were significantly downregulated upon loss of CTCF occupancy either at CTCF/BORIS or CTCF-only sites, while all upregulated pathways were not related to spermatogenesis and were equally enriched independent of CTCF and BORIS occupancy.

DEGs in CM testis were strongly correlated with the enrichment of RNAPII and H3K4me3 histone modification as shown by ChIP-seq in germ cells isolated from WT and CM testes (Supplementary Figure 13b). To integrate the transcriptomic and epigenomic datasets, DEGs were separated into three groups: down-regulated, up-regulated, and unchanged transcription for 
WT vs CM, and tag density plots of RNAPII and H3K4me3 ChIP-seq were generated for each group. The downregulation or upregulation of DEGs was well correlated with tag density for RNAPII and H3K4me3 around the TSS (Supplementary Figure 13b). Although these results present a coherent interpretation of both RNA-seq signal and RNAPII or H3K4me3 occupancy at the promoters of DEGs affected by lost CTCF and BORIS binding in CM testis, the loss of CTCF binding is unlikely to explain all observed transcriptional changes in CM testis. We cannot exclude the possibility that the deregulation of some genes in CM testis with no loss of CTCF occupancy in the promoter regions is related to small changes in germ-cell populations due to the heterogeneity found in CM testis (Supplementary Figure 1d).

Taken together, our findings demonstrate that both CTCF and BORIS activate the transcriptional program of spermatogenesis in male germline. Therefore, the loss of CTCF and BORIS occupancy specifically at CTCF/BORIS sites at the promoters of DEGs is likely the main contributing factor to the sterility of the $\mathrm{CM}$ male mice.

\section{"Toxic" KIT persistence beyond spermatogonia in the absence of CTCF and BORIS binding}

As mentioned above, transcriptomic analysis showed that a minority of genes was downregulated in CM testes. For example, Fads1 and Ankrd52 were highly upregulated in CM testes compared to WT upon loss of CTCF and BORIS occupancy in their promoter regions (Fig.6e, f). Although GO analysis suggests indirect effects on some of these genes (Fig.6d), the resulting overexpression of others could contribute to the sterility phenotype. In general, for other highly DEGs, the loss of specific CTCF/BORIS binding sites resulted in a severe deregulation of their transcription (Supplementary Figure 12b,c).

Among the genes affected in CM testis, one gene of particular biological importance was upregulated: the proto-oncogene Kit, a member of the tyrosine kinase receptor family that is crucial for the survival, proliferation, and maturation of primordial germ cells ${ }^{61}$. Although Kitdeficient mice die in the first days of postnatal life, some mutations in Kit result in viable but sterile male mice ${ }^{61}$. In testis, Kit is expressed in two isoforms: before meiosis, it is expressed as a fulllength transcript (Kit - Kit FL); after meiosis, it is expressed as a truncated isoform (Kit TR $)^{61}$. KIT is routinely used as a marker of differentiating spermatogonia, as Kit expression is strictly restricted to these cells, and its expression is necessary for triggering the differentiation process in the noncommitted spermatogonia stem cells ${ }^{61}$. Persistence of high-level KIT could potentially disrupt downstream differentiation of the developing germ cells, as an earlier study showed that constant expression of a Kit mutant in spermatids caused malformed mature spermatozoa ${ }^{62}$. 
Incidentally, the Kit promoter contains a CTCF/BORIS heterodimeric site and, in CM testis, the loss of both CTCF and BORIS occupancy resulted in a significant Kit upregulation (Fig.7a, Supplementary Figure 14a). Furthermore, qRT-PCR validation showed that Kit FL transcript expression was increased at least 12-fold in CM testis (Fig.7b), but the expression of Kit TR isoform was unaffected (Fig.7c). An increase in the overall level of Kit in CM testis was also detected by immunoblotting (Fig.7d). Accordingly, Gene Set Enrichment Analysis (GSEA) showed significant upregulation of the 52-gene KIT pathway, with a normalized enrichment score (NES) of 1.52 and a nominal p-value of 0.024 (Supplementary Figure 14b). Thus, Kit belongs to a group of DEGs that become upregulated in CM testis upon depletion of both CTCF and BORIS proteins in CM testis.

To determine whether the induction of Kit expression was a direct result of CTCF and BORIS simultaneous depletion, we first narrowed down the promoter-proximal region bound by CTCF and BORIS in WT testes to the first intron of mouse Kit, approximately 800 bp downstream of the TSS (Fig.7a). This region was previously identified as an essential regulatory element for Kit expression ${ }^{63}$. Second, by gel-shift assays, we confirmed that both CTCF and BORIS bound equally well to the Kit regulatory region (Fig.7e). The sequence of this region encompassed two 20bp-CTCF consensus sites separated by a 31-bp sequence (Fig.7f), thus belonging to the $2 x C T S$ class described previously ${ }^{33}$. This result is in good agreement with the simultaneous occupancy of CTCF and BORIS proteins at the same binding region (Fig.7a). The presence of a 2xCTS under one ChIP-seq peak also explains the double shift of DNA-protein complex observed by the gel-shift experiments using the 11ZF CTCF domain for the binding analysis (Fig.7e, double arrow). Thus, CTCF and BORIS co-binding to the promoter region of Kit directly represses Kit transcription during spermatogenesis.

Comparison of gene regulation among evolutionarily distant genomes permits identification of evolutionary conserved mechanisms in gene regulation. To investigate whether the mechanism of KIT regulation is conserved in humans, we analyzed KIT expression in the BORIS-positive human erythroleukemia cancer cell line, K562. ChIP-seq mapping of CTCF and BORIS occupancy in $\mathrm{K} 562$ cells $^{33}$ showed that the same region of the Kit locus is occupied by both CTCF and BORIS (Fig.7g). The alignment of mouse and human sequences under the CTCF/BORIS ChIP-seq peaks showed that both CTCF binding sites are conserved, confirming an evolutionary importance of CTCF/BORIS binding for KIT transcription (Fig.7f). To analyze whether CTCF and BORIS binding can also regulate KIT transcription in human cells, we knocked down BORIS expression in K562 cells using the zinc finger nuclease approach (Fig. 7i). Upon 
BORIS downregulation, KIT expression was upregulated at least 4-fold compared to parental K562 cells (Fig.7h, i), suggesting the direct involvement of CTCF and BORIS binding in the repression of Kit expression in humans as well as in mice.

As mentioned above, KIT expression is normally restricted to primary spermatogonia, where KIT is necessary to trigger the differentiation process in the noncommitted spermatogonia stem cells ${ }^{61}$. We postulated that the persistence of KIT expression beyond spermatogonia could disrupt downstream differentiation of the developing germ cells and lead to the male sterility, as it has been demonstrated in an earlier study ${ }^{62}$. Indeed, immunohistochemical analyses of KIT in 3-month-old testes (Fig.7j) showed an abnormal staining pattern of KIT in CM testis distinct from that in WT, CTCF HET and BORIS KO mice. In CM testis, in addition to spermatogonia staining, spermatocytes and round spermatids were also highly positive for KIT (Fig.7j). Therefore, the increase of KIT expression observed in CM testis can be explained by an atypical upregulation of KIT in meiotic and post meiotic cells (Fig.7j), explaining the abnormal sperm phenotypes such as loose and expanded sperm heads and aberrant flagella that are partially or completely coiled around the head (Fig.1b, S1c). We conclude that loss of BORIS and haploinsufficiency of CTCF in CM testis cause both downregulation of spermatogenesis-specific genes (Fig.6) and a "toxic" upregulation of inappropriate genes such as Kit, thereby explaining the severe sterility phenotype.

\section{DISCUSSION}

Here we investigated a longstanding question on the mode of functional interaction between CTCF and BORIS in male germline. Previously, while BORIS was known to be expressed only in male germ cells, BORIS-null mice inexplicably have only minimal germ cell defects $^{29,31,32}$. Upon generating mice with varied CTCF and BORIS levels, we uncovered that CTCF can partially compensate for BORIS loss, and when CTCF levels are also reduced, nearly complete loss of fertility occurs. That phenotype evidently stems from the necessity of CTCF and BORIS to heterodimerize at the key 2xCTSes genome wide. Indeed, our analysis shows that a large number of spermatogenesis controlling genes became repressed in CM testis. Such genes typically have heterodimeric CTCF/BORIS binding sites in their promoters and appear to be directly affected in the CM genotype (Figs. 5d, 6e, 7a, g, and Supplementary Figure 12a). On the other hand, some genes with heterodimeric CTCF/BORIS sites gain expression. The most intriguing among them is Kit, which uncoordinated persistence during germ cell development results in severe morphological and functional abnormalities (Fig. 7). We propose that the complete sterility of $\mathrm{CM}$ male mice results from both a failure to activate spermatogenesis-specific genes and the inability to repress pre-meiotic genes in a stage-specific manner. These findings 
further confirm that CTCF/BORIS heterodimer can be both a transcriptional activator and a repressor at the same time.

Rather unexpectedly, we also showed that CTCF and BORIS cooperation was required for the proper progression through meiosis I. Although the partial depletion of either CTCF in CTCF HET mice or full loss of BORIS in BORIS KO mice had underwhelming impact on male germ cell development, their combined deficiency in CM mice resulted in complete male sterility. The reduced cellularity of seminiferous tubules and the increased apoptosis in meiotic cells of CM testis point to defects in meiotic progression, which could lead to only a small fraction of germ cells progressing to the spermiogenesis stage. Indeed, CM spermatocytes showed an abnormal SC with atypical SYCP3 aggregates at some paired chromosomes and the persistence of unrepaired DSB beyond early pachynema, two processes found to be related in several studies $^{53,64}$. The establishment of the SC itself depends on several factors, including ones that are specific not only for meiotic cells: the formation of DNA loops mediated by somatic and testisspecific cohesin subunits ${ }^{65}$, the pairing of autosomal chromosomes ${ }^{66}$, and the stage-sensitive repair of $\mathrm{DSB}^{53,67}$. Incidentally, each of these processes has been known to be associated with CTCF function in somatic cells ${ }^{68-72}$. However, now we show that CTCF alone is insufficient to mediate similar processes in germ cells, and that a cooperation between CTCF and BORIS is required. Our results suggest at least three not mutually exclusive scenarios to explain how a reduction of CTCF expression in the absence of BORIS might lead to defects and/or delays in meiotic progression observed in CM mice. First, CTCF regulates the 3D architecture of eukaryotic genomes by blocking cohesin-mediated extrusion of chromatin loops, anchoring cohesin to CTCF binding sites ${ }^{46,73-76}$. In CM mice, CTCF occupancy was lost at thousands of genomic regions which could potentially lead to the loss of cohesin occupancy, affecting the formation of meiosis-specific DNA loops, essential for proper recombination during meiosis. Second, CTCF has been reported to be involved in the pairing of $\mathrm{X}$-chromosomes during the $\mathrm{X}$-inactivation in female somatic cells ${ }^{68,77}$. As the pairing of autosomes is a prerequisite for SC formation, the incomplete pairing of autosomes due to the loss of CTCF occupancy may lead to some abnormalities. Third, CTCF facilitates DSBs repair via interacting with some DNA repair factors such, as MRE11, CtIP, and Rad51 $1^{69,70}$. Specifically, the depletion of CTCF profoundly impairs the homologous recombination pathway of DSB repair by blocking CtIP recruitment to repair foci ${ }^{70}$. Thus, the persistence of DSBs beyond early pachynema in CM testis could be related to the deficiency of CTCF. The abnormal formation of the synaptonemal complex was already observed in BORIS KO mice ${ }^{32}$, but to a much lesse extent (20\% affected cells in BORIS KO versus $40 \%$ in $\mathrm{CM}$ testis), suggesting a direct 
involvement of BORIS in SC formation and the existence of a compensatory mechanism relying on the availability of CTCF.

Most importantly, our results provide compelling evidence that CTCF and BORIS cooperatively control the spermatogenesis-specific transcription program. Indeed, it can be concluded, that the infertility of CM mice is primarily due to a disruption of the germ cell specific transcription, with a pervasive deregulation of a substantial number of genes with known roles in spermatogenesis and sperm development. Undoubtedly, the loss of CTCF and BORIS occupancy at the promoter regions is the main driver of this transcriptional deregulation, especially at the 2xCTS binding sites normally occupied by both CTCF and BORIS. Such a requirement for the 2xCTS-driven CTCF and BORIS heterodimerization in transcriptional regulation of germline development is vividly illustrated by the two testis-specific genes, Tsp50 and Ga/3st1, previously found to be directly regulated by BORIS ${ }^{29,31,32}$. Both genes are essential for spermatogenesis ${ }^{78,79}$, and their downregulation upon Boris deletion can account for the bulk of subfertility phenotype of BORIS KO mice. The remaining expression of Tsp50 and Gal3st1 in BORIS KO mice may be the result of a functional compensation by CTCF for the loss of BORIS. Such a compensatory role for CTCF is consistent with the strikingly severe downregulation of Tsp50 and Gal3st1 expression in CM mice. However, we also demonstrated that in CM mice the Kit mRNA and protein were aberrantly accumulated in meiotic and post meiotic cells, where Kit is normally not expressed. While the depletion of either CTCF or BORIS led to a slight but not significant upregulation of Kit expression as compared to WT, the CM mice showed a dramatic upregulation of Kit expression by more than 12-fold. Similarly, a variety of other spermatogenesis-essential genes (including Fert2, Tssk3, Syse2, Tcp11, Prkar2a) followed the same pattern: depletion of either CTCF or BORIS only slightly impaired gene expression, but simultaneous ablation of CTCF and BORIS lead to the unexpectedly dramatic deregulation of these target genes. Thus, our data provide convincing evidence of functional cooperation between CTCF and BORIS in male germ cell development. Taking together the male germline restricted pattern of BORIS expression from sharks to mammals ${ }^{26,28}$ and the functional cooperation of CTCF and BORIS at the promoters of germ-cell specific genes, we would suggest that CTCF and BORIS heterodimerization is evolutionary more adapted in regulation of many germ-cell specific transcripts compared to CTCF homodimerization.

In addition to the heterodimerization between CTCF and BORIS on the protein-to-protein level, we also demonstrated, for the first time, that BORIS directly binds the Ctcf promoter and stimulates Ctcf expression in male germ cells. This was especially apparent in the context of Ctcf 
heterozygosity, where CTCF levels were reduced 75\% upon Boris deletion compared to WT testis. As a result of such a severe CTCF downregulation in CM testis, CTCF occupancy was lost at thousands of genomic regions, being more dramatic at $2 x C T S e s$ than at $1 \times C T S e s$. This observation also raises an important mechanistic question: why CTCF occupancy is preferentially lost in the regions bound by both CTCF and BORIS? Based on the data presented in this study and on the published studies, we propose a model to explain the non-additive phenotype of CM mice in comparison to WT, CTCF HET and BORIS KO mice (Supplementary Figure 15). This model is based on the well documented fact that CTCF can exist in the cells in the form of monomers, homodimers, and heterodimers with BORIS ${ }^{33,45,80}$. Furthermore, the transcriptional outcome of CTCF and BORIS heterodimerization is different from CTCF homodimerization at the $2 x$ CTSes $^{33,43,45}$, as the two paralogs recruit different protein-partners due to their divergence in the $\mathrm{N}$ - and $\mathrm{C}$-termini ${ }^{35}$. When CTCF and BORIS are expressed together in male germ cells, they preferentially form heterodimers at the active promoters and enhancers. This could indicate that CTCF/BORIS heterodimers are more stable and effective at the 2xCTSes as compared to CTCF homodimers at the same sites. The ectopic BORIS expression in BORIS-negative cells, provides a telling clue about BORIS KO phenotype in mice. Indeed, upon such expression, 2xCTSes were preferentially co-bound by both CTCF and BORIS instead of CTCF homodimers ${ }^{33}$. Therefore, in a reverse situation in BORIS KO germ cells, it would be logical to expect that CTCF homodimers might physically replace and compensate for the loss of CTCF-BORIS heterodimers due to sufficient supply of CTCF. However, as CTCF protein level in CM testis is so severely reduced, it is likely that such low level of CTCF may be insufficient to form stable homodimers at the 2xCTSes vacated by BORIS. However, the reduced level of CTCF may be sufficient for CTCF monomers to fill the $1 \times$ CTSes. In support of this idea, an acute depletion of CTCF using the auxin-induced degron system showed that the CTCF-binding affinity was significantly reduced at the $2 \times C T S e s$ that require CTCF homodimer binding as compared to $1 \times$ CTSes to which CTCF monomers bind ${ }^{81}$. It is also possible that the preferential additional loss of CTCF occupancy at 2xCTSes could be driven by downregulation of some cofactors required for CTCF co-binding or by a biological selection of surviving germ cells. As a result, upon loss of CTCF occupancy at the $2 \times C T S e s$, the transcription of target genes is severely affected in $\mathrm{CM}$ testis (See proposed model in Supplementary Figure 15).

We showed that the sterility of CM mice was associated with low sperm count, impaired morphology, and retarded motility of mature spermatozoa. None of these major contributors to male infertility were prominent in CTCF HET or BORIS KO mice. Similar sperm abnormalities and complete sterility were described for Ctcf-conditional knockout in pre-leptotene spermatocytes ${ }^{82}$. 
There, the Ctcf knockout affected the incorporation of protamine-1 into chromatin and histone retention in mature spermatozoa ${ }^{82}$. During spermiogenesis, protamines replace histones to permit chromatin compaction in the sperm head ${ }^{83}$. However, not all histones are replaced, some genomic regions retain histones to facilitate the transcription of genes essential for early zygotic activation 84-87. We recently showed that the regions that escape histone-to-protamine replacement are frequently associated with $2 x$ CTSes $^{33}$. Similarly, CTCF has been reported to be associated with the regions of retained histones in sperm ${ }^{84-86}$. As the loss of CTCF occupancy in CM testis preferentially occurred at 2xCTSes, it is likely that the loss of CTCF binding affected the retention of histones at these sites. If so the loss of CTCF binding would lead to an abnormal sperm owing to the accelerated histone depletion, as was described in the Ctcf-conditional knockout ${ }^{82}$.

Our analysis of RNA-seq data of four mouse genotypes suggested Kit as a candidate gene responsible for CM sperm abnormalities. Indeed, it has been reported before that the expression of a constitutively active Kit mutant in spermatids leads to malformations of mature spermatozoa, both in the head and in flagellum ${ }^{62}$. The Kit mutant phenotype is strikingly reminiscent of the phenotype of mature spermatozoa in $\mathrm{CM}$ mice, suggesting that the aberrantly high expression of KIT in CM spermatids may contribute to the abnormal sperm morphology observed in CM mice.

The present study strongly supports the conclusion of functional cooperation between CTCF and BORIS proteins in the regulation of normal male germ cell development. However, the contributions of CTCF and BORIS to spermatogenesis are not equivalent, as the conditional Ctcf knockout results in the complete male mice sterility, while Boris knockout leads to a subfertility phenotype only. Nonetheless, as we show here there are several processes where both CTCF and BORIS are necessary for a normal development, including the regulation of structural chromatin processes in meiosis, the control of spermatogenesis-specific gene transcription and the involvement in sperm genome organization.

The discovery of a well-tuned balance of two paralogous proteins in normal germ cell development contributes to our understanding of the role of aberrant BORIS expression in cancer development and progression. We previously showed that the aberrant BORIS expression in cancer cells is associated with the execution of a germline-like transcription program through CTCF and BORIS heterodimerization at promoter regions of cancer-testis genes ${ }^{33,88}$. Moreover, ectopic BORIS expression in normal somatic cells has been shown to activate multiple germlinespecific transcripts $29,33,89,90$. Thus, by uncovering the vital roles of both CTCF and BORIS for the transcriptional program of normal spermatogenesis, we now can better understand the consequences of aberrant BORIS expression in cancers. 


\section{METHODS}

\section{Mice and germ cells preparations.}

Boris and Ctcf knockout mouse models have been previously described ${ }^{22,32}$. The compound mutant, Ctcf $^{+/}$Boris $^{-/}$(CM), mice were generated from mating $\mathrm{Ctcf}^{+-}$Boris $^{+/+}$(CTCF HET) females and Ctct $^{++-}$Boris $^{--}$(BORIS KO) males. All mice were genotyped by PCR, as described ${ }^{22,32}$. The use of mice in this study was approved by the NIAID Animal Care and Use Committee under protocol LIG-15. The animals were maintained in the animal facility on a 12-h reverse light/dark cycle and provided with food and water ad libitum. The animal facility was maintained at a temperature of $21^{\circ} \mathrm{C}$ with $50-60 \%$ humidity.

For germ cells preparations, decapsulated testes of 3-month old mice were treated with $1 \mathrm{mg} / \mathrm{ml}$ of collagenase (Millipore/Sigma) to digest interstitial tissue and release single tubules in suspension. The tubules were next treated with $0.5 \mu \mathrm{g} / \mathrm{ml}$ trypsin (Millipore/Sigma) to generate a germ cells enriched single cells suspension. The supernatants were then passed through $100 \mu \mathrm{m}$ cell strainer (Corning) to discard tissue debris and washed with PBS. In order to obtain a better cell representation of the phenotypes observed, we included 4 different mice in each germ cells preparation.

Spermatocytes and round spermatids were purified by centrifugal elutriation as previously described ${ }^{25}$ followed by flow cytometric sorting of cells stained with Vybrant DyeCycle Green (Invitrogen, Carlsbad, CA) to obtain cell fractions with high purity. Briefly, decapsulated testes were treated with $1 \mathrm{mg} / \mathrm{ml}$ collagenase followed by treatment with $0.5 \mu \mathrm{g} / \mathrm{ml}$ trypsin, and the dissociated cells were used for centrifugal elutriation. Partially purified spermatocytes and round spermatids fractions were incubated with $10 \mu \mathrm{M}$ Vybrant DyeCycle Green for 30 min at $32^{\circ} \mathrm{C}$ followed by 4,6-diamidino-2-phenylindole (DAPI) staining. Cells were then sorted on a FACS-Aria (Becton Dickinson) to purify spermatocytes and round spermatids. DAPI-positive dead cells were eliminated. The purity of cells was confirmed by flow cytometry analysis of DNA content using sorted cells and by cell morphology analysis. The purity of round spermatids fraction was estimated to be approximately $93.7 \% \pm 2.3 \%$. The purity of spermatocytes was estimated to be approximately $81.5 \pm 3.7 \%$, based on the immunostaining with anti-SYCP3 abs. The expression of genes specific for spermatocytes and round spermatids was confirmed by RNA-seq. The list of marker genes for the specific stages of spermatogenesis was used from Shiota et al., $2018^{59}$. 
K562 cell line was grown in Dulbecco's modified Eagle medium (DMEM) supplemented with 10 $\%$ fetal calf serum and penicillin-streptomycin. K562 BORIS Knockout cell line was generated previously by us $^{33}$.

\section{Sperm analysis.}

The cauda epididymis of 3 month-old mice was dissected and cut into small pieces in PBS and incubated at $37 \mathrm{C}$ for 15 min allowing the release of spermatozoa to the medium. Sperm number and motility was analyzed in an IVOS system (Hamilton Thorne). To analyze sperm morphology, samples were washed with PBS, fixed in 4\% paraformaldehyde and smeared onto glass slides. Spermatozoa on air-dried slide were stained with hematoxylin.

\section{Histological analysis, immunohistochemistry and spermatocyte surface spread staining.}

Testes and cauda epididymis were fixed with $10 \%$ formalin and embedded in paraffin. Fivemicrometer sections were prepared for histological analysis and stained with hematoxylin-eosin. Terminal deoxynucleotidyltransferase-mediated dUTP-biotin nick end labeling (TUNEL) staining was performed using the DeadEnd colorimetric TUNEL system (Promega, Madison, WI) according to the manufacturer's protocol. TUNEL-positive cells in 30 tubules were counted to quantify apoptotic cells in testes. Three mice were examined for each genotype.

Surface spreads of spermatocytes were prepared as previously described ${ }^{44}$. Slides were incubated with $0.1 \%$ triton X-100 in PBS for 30 minutes at room temperature and washed 3 times in PBS for 30 minutes, blocking solution (5\% BSA) was added, and slides were incubated for 1 hour in a humid chamber at room temperature. Primary antibodies, rabbit anti- $\mathrm{y}-\mathrm{H} 2 \mathrm{AX}$, (ser189) (1:200, Millipore, \#07-164), mouse anti-SYCP3 (1:50, Santa Cruz ,sc-74569), and rabbit polyclonal-SYCP1 (1:1000, Abcam, \#15090), were diluted in blocking buffer, and slides were incubated overnight in a humid chamber at $4^{\circ} \mathrm{C}$. After washing, secondary antibodies were used at a 1:200 dilution for 1 hour at room temperature, donkey anti-rabbit AlexaFluor488 (A-21206, Thermo Fisher Scientific), goat anti-rabbit AlexaFluor 615 Texas red (T-2767 Thermo Fisher Scientific), donkey anti-mouse AlexaFluor488 (A-21202, Thermo Fisher Scientific), and goat anti-mouse AlexaFluor 615 Texas red (T-6390, Thermo Fisher Scientific). The slides were washed and allowed to dry and mounted with Vectashield Mounting Medium with DAPI (Vector Laboratories). Fluorescent images were acquired using a confocal laser-scanningmicroscope (LSM 780 Carl Zeiss).

For Kit Immunohistochemistry, sections were boiled for $10 \mathrm{~min}$ in $10 \mathrm{mM}$ sodium citrate buffer $(\mathrm{pH}$ 6.0), treated with $1 \% \mathrm{H}_{2} \mathrm{O}_{2}$, blocked with $5 \%$ goat serum, and incubated with antibodies against Kit (1:200 anti Kit AF1356 R\&D Systems). The antigen-bound primary antibodies were detected 
using 1:500 dilution of biotinylated anti-goat IgG antibody (BA-5000-1.5, Vector Laboratories) and avidin-conjugated peroxidase (Vector Laboratories). In all immunohistochemical analyses, peroxidase activity was visualized by using 3,3'-diaminobemnzidine as a substrate. Substitution of the primary antibodies with nonimmune IgG was performed as negative control. After staining, sections were counterstained with hematoxylin (MilliporeSigma).

\section{RNA-seq experiments.}

For RNA-seq from the whole testis, rRNA-depleted RNA from 3 independent germ cells preparations described as indicated before (4 mice in each preparation) was prepared using the RiboMinus Eukaryote System v2 kit (Life Technologies) according to the manufacture's recommendations. $500 \mathrm{ng}$ of rRNA-depleted RNA was used for library preparation using the lon Total RNA-seq v2 kit (Life Technologies). The enriched libraries were diluted to a final concentration of $11 \mathrm{pM}$ and subjected to sequencing from a single end in a lon Proton Sequencer (Life Technologies). FASTQ files were mapped to the UCSC mouse reference genome (build mm9) using two-step alignments. First, the reads were aligned with TopHat2 ${ }^{91}$. Second, the unmapped reads from the first step were extracted and aligned with Bowtie $2^{59}$ with -local mode and --very-sensitive-local option. For RNA-seq from spermatocytes and round spermatids, 3 replicates were used for each group, except for WT spermatocytes for which 2 replicates were used. Total RNA was extracted from cells using Trizol (Life Technologies) according to the protocol provided by the manufacturer. The RNA quality was assessed using an Agilent 2100 Bioanalyzer. The RNA sequencing library preparation and sequencing procedures were carried out according to Illumina protocols with minor modifications. Briefly, poly $(A)$-mRNA was purified from $5 \mu \mathrm{g}$ of RNA with streptavidin-coated magnetic beads. After chemical fragmentation, mRNA fragments were reverse-transcribed and converted into double-stranded cDNA. Following end repair and A-tailing, paired-end adaptors were ligated to the ends of the DNA fragments. The ligated products were amplified with 18 cycles of PCR followed by purification using AMPure beads (Beckman Coulter). The enriched libraries were diluted to a final concentration of $5 \mathrm{nM}$. Each sample was subjected to 75 cycles of sequencing from a single end in an Illumina HiSeq2000 Sequencer. FASTQ files were mapped to the UCSC mouse reference genome (build mm9) using STAR ${ }^{92}$ with default settings. Normalized gene counts and differentially expressed genes (DEG) were obtained with the $R$ package DESeq2 ${ }^{45}$. The genes with a p-adjusted $<0.001$ and fold change $>2$ were considered as significant DEGs. To identify round spermatid marker genes (Supplementary Figure 9), DEGs between spermatocytes and round spermatids in WT mice were identified using DESeq2 and very stringent conditions to guarantee that we are identifying true markers ( $p$-adjusted-value $<1 \mathrm{E}-06$ and log2Fold change $>4$ ). Then, the 
differentially expressed genes in CM whole testis were removed from the list for a total of 833 upregulated genes in WT round spermatids compared to WT spermatocytes (post-meiotic markers not affected in CM testis). Additionally, we have used the marker genes for round spermatids from in Shiota et al., $2018^{59}$.

Gene Ontology analysis was performed in DAVID Bioinformatics Resources ${ }^{93}$. Gene Set Enrichment Analysis (GSEA) was performed using the GSEA v4.1.0 software ${ }^{33}$ (http://software.broadinstitute.org/gsea/index.jsp) and the following Molecular Signatures Database (MSigDB): the Hallmark gene sets $(\mathrm{H})$, the Curated gene sets (C2) and the Ontology gene sets (C5) (http://www.gsea-msigdb.org/gsea/msigdb/collections.jsp). GSEA of the Kit pathway was performed using the Kit Pathway gene set containing 52 genes (https://www.gseamsigdb.org/gsea/msigdb/cards/PID_KIT_PATHWAY). We chose the t-test as metric for ranking genes and 1,000 gene set permutations were used to generate a null distribution for the enrichment score, which was used to yield a normalized enrichment score (NES) for the gene set.

\section{ChIP sequencing.}

Two germ cell enriched preparations were obtained as described above. 60 million cells were crosslinked with $1 \%$ formaldehyde for $10 \mathrm{~min}$ at room temperature, followed by quenching with $125 \mathrm{mM}$ glycine for 10 min, washed twice with 1x PBS, and resuspended in ChIP lysis buffer (150 mM NaCl, 1\% Triton X-100, 0.1\% SDS, 20 mM Tris-HCl pH8.0, 2 mM EDTA). Chromatin was sheared to an average length of $200-500$ bp using a Bioruptor sonicator. After overnight incubation with DiaMag magnetic beads (Diagenode, Inc.) and antibodies, precipitated chromatin was then washed, decrosslinked, and digested with proteinase K (Millipore/Sigma). The resulting DNA was purified using phenol/chloroform/isoamyl alcohol. DNA concentration was assessed with the Quant-it PicoGreen dsDNA kit (Life Technologies) and 5-10 ng was used to generate sequencing libraries. ChIP DNA was amplified using the TruSeq ChIP Sample Preparation Kit (Illumina, Inc., USA). Briefly, the immunoprecipitated material was end-repaired, A-tailed, ligated to the sequencing adapters, amplified by 15 cycles of PCR, and size selected (200-400 bp) followed by single end sequencing on an Illumina Genome Analyzer according to the manufacturer's recommendations. For spermatocytes ChIP-seq, $\sim 20$ million of purified cells were crosslinked with $1 \%$ formaldehyde for $10 \mathrm{~min}$ at room temperature, followed by the same procedure as described for germ cells extracted from the whole testes. For round spermatids CTCF ChIP-seq, we reanalyzed the data previously produced and described by us (GSE70764, https://www.ncbi.nlm.nih.gov/geo/query/acc.cgi?acc=GSE70764) ${ }^{33}$. For BORIS ChIP, an antibodies previously characterized by us were used ${ }^{29,32,94}$. For CTCF, an equimolar mix of CTCF 
C-terminus commercial antibody were used (anti-CTCF B-5(sc-271514) and C-20 (sc-15914)) from Santa Cruz; anti-CTCF (D31H2) from Cell Signaling: anti-CTCF (07-729) from Millipore: antiCTCF (70303) from Abcam: anti-CTCF (A300-543A) from Bethyl and anti-CTCF (NB500-177 and NB500-194) from Novus. For Polymerase II and H3K4me3 ChIP-seq anti-Pol2-4H8 (Abcamab5408) and anti-H3K4me3 (Abcam-ab8580) were used.

Sequences generated by the Illumina Genome Analyzer (50bp reads) were aligned against the USCS mouse reference genome (build mm9) using Bowtie2 program (http://bowtiebio.sourceforge.net). The alignment was performed with default parameters using the $-\mathrm{m} 5$ option. Peaks were called using Model-based Analysis for ChIP-Seq (MACS) and (MACS2) 95 using default parameters. For Polymerase II and H3K4me3 the options --nomodel --extsize 74 were included. After MACS, we applied Peak Splitter algorithm (part of MACS) to call sub-peaks, summit of peaks and improve peak resolution. The ChIP-seq data were visualized using Integrative Genomics Viewer (IGV) (https://www.broadinstitute.org/software/igv). The peak overlaps between CTCF and BORIS ChIP-seq data sets were carried out with BedTools Suite (http://bedtools.readthedocs.org). We defined peaks as overlapping if at least $1 \mathrm{bp}$ of reciprocal peaks intersect (CTCF/BORIS), the remaining peaks were defined as non-overlapping (CTCFonly and BORIS-only). The normalized tag density profiles were generated using BedTools coverage option from BedTools Suite, normalized to the number of mapped reads, and plotted by Microsoft Excel. The heatmaps were generated using seqMINER 1.3.3 platform ${ }^{96}$. We used either K-means ranked or linear method for clustering normalization. The summit of either CTCF or BORIS peaks were extended as indicated in the figures. seqMINER was also used to generate the average profiles of read density for different clusters. The annotatePeaks.pl feature of HOMER (http://homer.salk.edu/homer/index.html) was used to generate average plots around CTCF and TSS sites with 10 bp resolution. Default normalization (scaling of tag directories to 10 million reads) was used. Genomic distributions of CTCF and BORIS peaks were identified with the annotatePeaks.pl feature of HOMER(v4.11). CTCF motif analysis were done with MAST (Motif Alignment and Search Tool, MEME suite v5.3.3).

\section{Western blot.}

Protein extracts were prepared by lysing germ cells isolated as describe above (RNA-seq section) or K562 cells in RIPA Lysis buffer (Millipore) containing 50 mM Tris- $\mathrm{HCl}$, pH 7.4, 1\% Nonidet P$40,0.25 \%$ sodium deoxycholate, $500 \mathrm{mM} \mathrm{NaCl}, 1 \mathrm{mM}$ EDTA, $1 X$ protease inhibitor cocktail (Roche Applied Science). Protein samples were resolved by SDS-PAGE, transferred to a PVDF membrane, and incubated with the indicated antibodies. Detections were performed using ECL 
reagents (Pierce). Kit protein was detected with 1:2000 anti c-kit (AF1356 R\&D Systems) and 1:2000 of anti-goat secondary antibody (sc2020, Santa Cruz). CTCF protein was detected with anti-CTCF B-5 (sc-271514, Santa Cruz) and 1:2000 of an anti-mouse secondary antibody (sc2005, Santa Cruz). Tubulin was detected using 1:5000 anti- $\alpha$ Tubulin B-7 (sc-5286, Santa Cruz) and 1:2000 of an anti-mouse secondary antibody (sc-2005, Santa Cruz). Images were quantified using ImageJ (v1.48) software and normalized to Tubulin.

\section{Electrophoretic mobility shift assay (EMSA).}

DNA fragments encompassing 200 bp long sequences derived from either CTCF and BORIS ChIP-seq peaks in Kit locus were synthesized by PCR using the following primers 5'CCACCCAACTCCGTTTTTTGCAC-3' and 5'-AACGAACACCGTGCGGCTGCAGAG-3'. The PCR products were confirmed by sequencing. EMSA was performed as previously described ${ }^{94}$. Briefly, PCR fragments were labeled using ${ }^{32} \mathrm{P}-\mathrm{y}$-ATP with T4 polynucleotide kinase (New England, Biolabs). Protein-DNA complexes were incubated for $1 \mathrm{~h}$ at room temperature in binding buffer containing $25 \mathrm{mM}$ Tris $\mathrm{pH} 7.4,0.1 \mathrm{mM} \mathrm{ZnSO}_{4}, 5 \mathrm{mM} \mathrm{MgCl}$, 5\% Nonidet P-40 in PBS, 0.25 mM 2-mercaptoethanol, $10 \%$ glycerol and $0.5 \mu \mathrm{g}$ of poly $\mathrm{dl}-\mathrm{dC}$. Protein-DNA complexes were separated from the unbound probe using $5 \%$ native polyacrylamide gels (PAAG) or $1.2 \%$ agarose gels run in $0.5 x$ Tris-borate-EDTA buffer. Full-length CTCF, full-length BORIS and CTCF 11 ZF domain were translated in vitro using the TnT Quick Coupled Transcription/Translation System (Promega).

\section{Real Time PCR.}

Total RNA was prepared using the RNeasy minikit (Qiagen, Valencia, CA). cDNA was prepared using the SuperScript III first-strand synthesis system (Invitrogen) according to the manufacturer's protocol. Real Time PCR was performed using the SYBR green PCR master mix (Applied Biosystems, Foster City, CA) and an 7900HT sequence detection system (Applied Biosystems). Expression levels were normalized with the housekeeping gene Gapdh. Primers used are listed in the Supplementary Data 6.

\section{Statistical analysis.}

In all figure legends, $\mathrm{n}$ represents the number of independent experiments conducted. Statistical analysis was performed using $\mathrm{R}$. Two-side unpaired t-test (confidence level 0.95) was used to calculate the $p$-value for all figures indicated with the exception of Fig1.e and Fig.1f where a twosided unpaired Mann-Whitney's test (confidence level 0.95) was used. Excat p-values are indicated in the figures or in the figure legends. In box plots, the lower and upper hinges 
correspond to the first and third quartiles, the middle line indicates the median. The upper whisker extends from the hinge to the largest value no further than $1.5^{*}$ IQR from the hinge (where IQR is the inter-quartile range, or distance between the first and third quartiles). The lower whisker extends from the hinge to the smallest value at most 1.5 * IQR of the hinge. In jitter charts (Fig.1a), the mean is indicated as a horizontal line.

\section{KEYWORDS}

CTCF, BORIS, CTCFL, spermatogenesis, meiosis, sterility

\section{ACKNOWLEDGMENTS}

We thank Dr. Susan Pierce and Dr. Louis Miller for support, critical reading of the manuscript, and helpful discussions. This work was supported by the Intramural Research Program of the National Institute of Allergy and Infectious Diseases (NIAID) (to V.V.L.) and the NIH (V.V.L.). The work of J.T.L. was supported by NIH grant R37-GM58839. A.V.S. was supported by the MOST National Key R\&D Program of China project number 2018YFA0106903. This study used the Office of Cyber Infrastructure and Computational Biology High Performance Computing cluster at NIAID, and high-performance computational capabilities of the Biowulf Linux cluster at NIH.

\section{COMPETING INTEREST}

The authors declare that they have no competing interests.

\section{AUTHOR'S CONTRIBUTIONS}

Conceived and designed the experiments: SR-H, EMP, DL, and VVL. Performed the experiments: SR-H, EMP, SK, CM-C and ALK. Analyzed the data: SR-H, EMP. Wrote the paper: SR-H, EMP, AVS, JTL, and VVL. All authors read and approved the final manuscript.

\section{DATA AVAILABILITY}

The authors declare that all data that support the findings of this study are available in this article and are provided as a Source File Data file or from the corresponding authors upon reasonable request. This study used data from the previously deposited dataset GSE70764 (https://www.ncbi.nlm.nih.gov/geo/query/acc.cgi?acc=GSE70764). The accession number for the ChIP-seq and RNA-seq data reported in this paper is GSE154249 (https://www.ncbi.nlm.nih.gov/geo/query/acc.cgi?acc=GSE154249). Molecular Signatures 
Database (MSigDB): the Hallmark gene sets $(\mathrm{H})$, the Curated gene sets $(\mathrm{C} 2)$ ( and the Ontology gene sets (C5) (http://www.gsea-msigdb.org/gsea/msigdb/collections.jsp). GSEA of Kit pathway was performed using the Kit Pathway gene set containing 52 genes (https://www.gseamsigdb.org/gsea/msigdb/cards/PID_KIT_PATHWAY).

\section{REFERENCES}

1. Phillips, J.E. \& Corces, V.G. CTCF: master weaver of the genome. Cell 137, 1194-211 (2009).

2. Rowley, M.J. \& Corces, V.G. Organizational principles of 3D genome architecture. Nat Rev Genet 19, 789-800 (2018).

3. Kim, T.H. et al. Analysis of the vertebrate insulator protein CTCF-binding sites in the human genome. Cell 128, 1231-45 (2007).

4. Shen, Y. et al. A map of the cis-regulatory sequences in the mouse genome. Nature 488, 116-20 (2012).

5. Smith, S.T. et al. Genome wide ChIP-chip analyses reveal important roles for CTCF in Drosophila genome organization. Dev Biol 328, 518-28 (2009).

6. Nuebler, J., Fudenberg, G., Imakaev, M., Abdennur, N. \& Mirny, L.A. Chromatin organization by an interplay of loop extrusion and compartmental segregation. Proc Natl Acad Sci U S A 115, E6697-E6706 (2018).

7. Fudenberg, G. et al. Formation of Chromosomal Domains by Loop Extrusion. Cell Rep 15, 2038-49 (2016).

8. Sanborn, A.L. et al. Chromatin extrusion explains key features of loop and domain formation in wild-type and engineered genomes. Proc Natl Acad Sci U S A 112, E6456-65 (2015).

9. Merkenschlager, M. \& Nora, E.P. CTCF and Cohesin in Genome Folding and Transcriptional Gene Regulation. Annu Rev Genomics Hum Genet 17, 17-43 (2016).

10. Baniahmad, A., Steiner, C., Kohne, A.C. \& Renkawitz, R. Modular structure of a chicken lysozyme silencer: involvement of an unusual thyroid hormone receptor binding site. Cell 61, 505-14 (1990).

11. Bell, A.C., West, A.G. \& Felsenfeld, G. The protein CTCF is required for the enhancer blocking activity of vertebrate insulators. Cell 98, 387-96 (1999).

12. Chao, W., Huynh, K.D., Spencer, R.J., Davidow, L.S. \& Lee, J.T. CTCF, a candidate transacting factor for X-inactivation choice. Science 295, 345-7 (2002).

13. Cuddapah, S. et al. Global analysis of the insulator binding protein CTCF in chromatin barrier regions reveals demarcation of active and repressive domains. Genome Res 19, 24-32 (2009).

14. Defossez, P.A. et al. The human enhancer blocker CTC-binding factor interacts with the transcription factor Kaiso. J Biol Chem 280, 43017-23 (2005).

15. Dixon, J.R. et al. Topological domains in mammalian genomes identified by analysis of chromatin interactions. Nature 485, 376-80 (2012).

16. Filippova, G.N. et al. CTCF-binding sites flank CTG/CAG repeats and form a methylationsensitive insulator at the DM1 locus. Nat Genet 28, 335-43 (2001). 
17. Kanduri, C. et al. Functional association of CTCF with the insulator upstream of the H19 gene is parent of origin-specific and methylation-sensitive. Curr Biol 10, 853-6 (2000).

18. Lobanenkov, V.V. et al. A novel sequence-specific DNA binding protein which interacts with three regularly spaced direct repeats of the CCCTC-motif in the 5'-flanking sequence of the chicken c-myc gene. Oncogene 5, 1743-53 (1990).

19. Rasko, J.E. et al. Cell growth inhibition by the multifunctional multivalent zinc-finger factor CTCF. Cancer Res 61, 6002-7 (2001).

20. Kung, J.T. et al. Locus-specific targeting to the $X$ chromosome revealed by the RNA interactome of CTCF. Mol Cell 57, 361-75 (2015).

21. Sun, S. et al. Jpx RNA activates Xist by evicting CTCF. Cell 153, 1537-51 (2013).

22. Moore, J.M. et al. Loss of maternal CTCF is associated with peri-implantation lethality of Ctcf null embryos. PLoS One 7, e34915 (2012).

23. Fedoriw, A.M., Stein, P., Svoboda, P., Schultz, R.M. \& Bartolomei, M.S. Transgenic RNAi reveals essential function for CTCF in $\mathrm{H} 19$ gene imprinting. Science 303, 238-40 (2004).

24. Soshnikova, N., Montavon, T., Leleu, M., Galjart, N. \& Duboule, D. Functional analysis of CTCF during mammalian limb development. Dev Cell 19, 819-30 (2010).

25. Kemp, C.J. et al. CTCF haploinsufficiency destabilizes DNA methylation and predisposes to cancer. Cell Rep 7, 1020-9 (2014).

26. Hore, T.A., Deakin, J.E. \& Marshall Graves, J.A. The evolution of epigenetic regulators CTCF and BORIS/CTCFL in amniotes. PLoS Genet 4, e1000169 (2008).

27. Loukinov, D.I. et al. BORIS, a novel male germ-line-specific protein associated with epigenetic reprogramming events, shares the same 11-zinc-finger domain with CTCF, the insulator protein involved in reading imprinting marks in the soma. Proc Natl Acad Sci U S A 99, 6806-11 (2002).

28. Kadota, M., Yamaguchi, K., Hara, Y. \& Kuraku, S. Early vertebrate origin of CTCFL, a CTCF paralog, revealed by proximity-guided shark genome scaffolding. Sci Rep 10, 14629 (2020).

29. Kosaka-Suzuki, N. et al. Transcription factor BORIS (Brother of the Regulator of Imprinted Sites) directly induces expression of a cancer-testis antigen, TSP50, through regulated binding of BORIS to the promoter. J Biol Chem 286, 27378-88 (2011).

30. Pugacheva, E.M. et al. The structural complexity of the human BORIS gene in gametogenesis and cancer. PLoS One 5, e13872 (2010).

31. Sleutels, F. et al. The male germ cell gene regulator CTCFL is functionally different from CTCF and binds CTCF-like consensus sites in a nucleosome composition-dependent manner. Epigenetics Chromatin 5, 8 (2012).

32. Suzuki, T. et al. Expression of a testis-specific form of Gal3st1 (CST), a gene essential for spermatogenesis, is regulated by the CTCF paralogous gene BORIS. Mol Cell Biol 30, 247384 (2010).

33. Pugacheva, E.M. et al. Comparative analyses of CTCF and BORIS occupancies uncover two distinct classes of CTCF binding genomic regions. Genome Biol 16, 161 (2015).

34. Klenova, E.M., Morse, H.C., 3rd, Ohlsson, R. \& Lobanenkov, V.V. The novel BORIS + CTCF gene family is uniquely involved in the epigenetics of normal biology and cancer. Semin Cancer Biol 12, 399-414 (2002). 
35. de Necochea-Campion, R. et al. Expression of the epigenetic factor BORIS (CTCFL) in the human genome. J Transl Med 9, 213 (2011).

36. Pugacheva, E.M. et al. CTCF mediates chromatin looping via N-terminal domaindependent cohesin retention. Proc Natl Acad Sci U S A 117, 2020-2031 (2020).

37. Nishana, M. et al. Defining the relative and combined contribution of CTCF and CTCFL to genomic regulation. Genome Biol 21, 108 (2020).

38. Martin-Kleiner, I. BORIS in human cancers -- a review. Eur J Cancer 48, 929-35 (2012).

39. Renaud, S. et al. Expression of the CTCF-paralogous cancer-testis gene, brother of the regulator of imprinted sites (BORIS), is regulated by three alternative promoters modulated by CpG methylation and by CTCF and p53 transcription factors. Nucleic Acids Res 35, 7372-88 (2007).

40. Simpson, A.J., Caballero, O.L., Jungbluth, A., Chen, Y.T. \& Old, L.J. Cancer/testis antigens, gametogenesis and cancer. Nat Rev Cancer 5, 615-25 (2005).

41. Loukinov, D. Targeting CTCFL/BORIS for the immunotherapy of cancer. Cancer Immunol Immunother 67, 1955-1965 (2018).

42. Soltanian, S. \& Dehghani, H. BORIS: a key regulator of cancer stemness. Cancer Cell Int 18, 154 (2018).

43. Hillman, J.C. et al. BORIS Expression in Ovarian Cancer Precursor Cells Alters the CTCF Cistrome and Enhances Invasiveness through GALNT14. Mol Cancer Res 17, 2051-2062 (2019).

44. Lobanenkov, V.V. \& Zentner, G.E. Discovering a binary CTCF code with a little help from BORIS. Nucleus 9, 33-41 (2018).

45. Debruyne, D.N. et al. BORIS promotes chromatin regulatory interactions in treatmentresistant cancer cells. Nature 572, 676-680 (2019).

46. Jung, Y.H. et al. Chromatin States in Mouse Sperm Correlate with Embryonic and Adult Regulatory Landscapes. Cell Rep 18, 1366-1382 (2017).

47. Creasy, D. et al. Proliferative and nonproliferative lesions of the rat and mouse male reproductive system. Toxicol Pathol 40, 40S-121S (2012).

48. Wu, Y. et al. Transcription Factor RFX2 Is a Key Regulator of Mouse Spermiogenesis. Sci Rep 6, 20435 (2016).

49. Zickler, D. \& Kleckner, N. Recombination, Pairing, and Synapsis of Homologs during Meiosis. Cold Spring Harb Perspect Biol 7(2015).

50. Lam, I. \& Keeney, S. Mechanism and regulation of meiotic recombination initiation. Cold Spring Harb Perspect Biol 7, a016634 (2014).

51. Kinner, A., Wu, W., Staudt, C. \& Iliakis, G. Gamma-H2AX in recognition and signaling of DNA double-strand breaks in the context of chromatin. Nucleic Acids Res 36, 5678-94 (2008).

52. Turner, J.M. Meiotic sex chromosome inactivation. Development 134, 1823-31 (2007).

53. Smagulova, F. et al. Suppression of genetic recombination in the pseudoautosomal region and at subtelomeres in mice with a hypomorphic Spo11 allele. BMC Genomics 14, 493 (2013).

54. Lyndaker, A.M. et al. Conditional inactivation of the DNA damage response gene Hus1 in mouse testis reveals separable roles for components of the RAD9-RAD1-HUS1 complex in meiotic chromosome maintenance. PLoS Genet 9, e1003320 (2013). 
55. Guo, J. et al. The adult human testis transcriptional cell atlas. Cell Res 28, 1141-1157 (2018).

56. Green, C.D. et al. A Comprehensive Roadmap of Murine Spermatogenesis Defined by Single-Cell RNA-Seq. Dev Cell 46, 651-667 e10 (2018).

57. Rivero-Hinojosa, S., Kang, S., Lobanenkov, V.V. \& Zentner, G.E. Testis-specific transcriptional regulators selectively occupy BORIS-bound CTCF target regions in mouse male germ cells. Sci Rep 7, 41279 (2017).

58. An integrated encyclopedia of DNA elements in the human genome. Nature 489, 57-74 (2012).

59. Shiota, H. et al. Nut Directs p300-Dependent, Genome-Wide H4 Hyperacetylation in Male Germ Cells. Cell Rep 24, 3477-3487 e6 (2018).

60. Makovski, A., Yaffe, E., Shpungin, S. \& Nir, U. Intronic promoter drives the BORISregulated expression of FerT in colon carcinoma cells. J Biol Chem 287, 6100-12 (2012).

61. Rossi, P., Sette, C., Dolci, S. \& Geremia, R. Role of c-kit in mammalian spermatogenesis. J Endocrinol Invest 23, 609-15 (2000).

62. Schnabel, D., Ramirez, L., Gertsenstein, M., Nagy, A. \& Lomeli, H. Ectopic expression of KitD814Y in spermatids of transgenic mice, interferes with sperm morphogenesis. Dev Dyn 233, 29-40 (2005).

63. Cairns, L.A. et al. Kit regulatory elements required for expression in developing hematopoietic and germ cell lineages. Blood 102, 3954-62 (2003).

64. de Vries, F.A. et al. Mouse Sycp1 functions in synaptonemal complex assembly, meiotic recombination, and XY body formation. Genes Dev 19, 1376-89 (2005).

65. McNicoll, F., Stevense, M. \& Jessberger, R. Cohesin in gametogenesis. Curr Top Dev Biol 102, 1-34 (2013).

66. Page, J. et al. Sex chromosomes, synapsis, and cohesins: a complex affair. Chromosoma 115, 250-9 (2006).

67. Keeney, S. Spo11 and the Formation of DNA Double-Strand Breaks in Meiosis. Genome Dyn Stab 2, 81-123 (2008).

68. $\mathrm{Xu}, \mathrm{N}$. , Donohoe, M.E., Silva, S.S. \& Lee, J.T. Evidence that homologous X-chromosome pairing requires transcription and Ctcf protein. Nat Genet 39, 1390-6 (2007).

69. Hilmi, K. et al. CTCF facilitates DNA double-strand break repair by enhancing homologous recombination repair. Sci Adv 3, e1601898 (2017).

70. Hwang, S.Y. et al. CTCF cooperates with CtIP to drive homologous recombination repair of double-strand breaks. Nucleic Acids Res 47, 9160-9179 (2019).

71. Sofueva, S. et al. Cohesin-mediated interactions organize chromosomal domain architecture. EMBO J 32, 3119-29 (2013).

72. Zuin, J. et al. Cohesin and CTCF differentially affect chromatin architecture and gene expression in human cells. Proc Natl Acad Sci U S A 111, 996-1001 (2014).

73. Parelho, V. et al. Cohesins functionally associate with CTCF on mammalian chromosome arms. Cell 132, 422-33 (2008).

74. Phillips-Cremins, J.E. et al. Architectural protein subclasses shape 3D organization of genomes during lineage commitment. Cell 153, 1281-95 (2013).

75. Vara, C. et al. Three-Dimensional Genomic Structure and Cohesin Occupancy Correlate with Transcriptional Activity during Spermatogenesis. Cell Rep 28, 352-367 e9 (2019). 
76. Wendt, K.S. et al. Cohesin mediates transcriptional insulation by CCCTC-binding factor. Nature 451, 796-801 (2008).

77. Abe, H. et al. The Initiation of Meiotic Sex Chromosome Inactivation Sequesters DNA Damage Signaling from Autosomes in Mouse Spermatogenesis. Curr Biol 30, 408-420 e5 (2020).

78. Zhang, Y. et al. Testis-specific sulfoglycolipid, seminolipid, is essential for germ cell function in spermatogenesis. Glycobiology 15, 649-54 (2005).

79. Zhou, L. et al. Knockdown of TSP50 inhibits cell proliferation and induces apoptosis in P19 cells. IUBMB Life 62, 825-32 (2010).

80. Yusufzai, T.M., Tagami, H., Nakatani, Y. \& Felsenfeld, G. CTCF tethers an insulator to subnuclear sites, suggesting shared insulator mechanisms across species. Mol Cell 13, 291-8 (2004).

81. Hyle, J. et al. Acute depletion of CTCF directly affects MYC regulation through loss of enhancer-promoter looping. Nucleic Acids Res 47, 6699-6713 (2019).

82. Hernandez-Hernandez, A., Lilienthal, I., Fukuda, N., Galjart, N. \& Hoog, C. CTCF contributes in a critical way to spermatogenesis and male fertility. Sci Rep 6, 28355 (2016).

83. Wang, T., Gao, H., Li, W. \& Liu, C. Essential Role of Histone Replacement and Modifications in Male Fertility. Front Genet 10, 962 (2019).

84. Arpanahi, A. et al. Endonuclease-sensitive regions of human spermatozoal chromatin are highly enriched in promoter and CTCF binding sequences. Genome Res 19, 1338-49 (2009).

85. Brykczynska, U. et al. Repressive and active histone methylation mark distinct promoters in human and mouse spermatozoa. Nat Struct Mol Biol 17, 679-87 (2010).

86. Erkek, S. et al. Molecular determinants of nucleosome retention at $\mathrm{CpG}$-rich sequences in mouse spermatozoa. Nat Struct Mol Biol 20, 868-75 (2013).

87. Luense, L.J. et al. Gcn5-Mediated Histone Acetylation Governs Nucleosome Dynamics in Spermiogenesis. Dev Cell 51, 745-758 e6 (2019).

88. Pugacheva, E.M. et al. The cancer-associated CTCFL/BORIS protein targets multiple classes of genomic repeats, with a distinct binding and functional preference for humanoid-specific SVA transposable elements. Epigenetics Chromatin 9, 35 (2016).

89. Vatolin, S. et al. Conditional expression of the CTCF-paralogous transcriptional factor BORIS in normal cells results in demethylation and derepression of MAGE-A1 and reactivation of other cancer-testis genes. Cancer Res 65, 7751-62 (2005).

90. Hong, J.A. et al. Reciprocal binding of CTCF and BORIS to the NY-ESO-1 promoter coincides with derepression of this cancer-testis gene in lung cancer cells. Cancer Res 65, 7763-74 (2005).

91. Kim, D. et al. TopHat2: accurate alignment of transcriptomes in the presence of insertions, deletions and gene fusions. Genome Biol 14, R36 (2013).

92. Shawlot, W., Vazquez-Chantada, M., Wallingford, J.B. \& Finnell, R.H. Rfx2 is required for spermatogenesis in the mouse. Genesis (2015).

93. Huang da, W., Sherman, B.T. \& Lempicki, R.A. Systematic and integrative analysis of large gene lists using DAVID bioinformatics resources. Nat Protoc 4, $44-57$ (2009). 
94. Pugacheva, E.M. et al. Familial cases of point mutations in the XIST promoter reveal a correlation between CTCF binding and pre-emptive choices of $\mathrm{X}$ chromosome inactivation. Hum Mol Genet 14, 953-65 (2005).

95. Zhang, Y. et al. Model-based analysis of ChIP-Seq (MACS). Genome Biol 9, R137 (2008).

96. Ye, T. et al. seqMINER: an integrated ChIP-seq data interpretation platform. Nucleic Acids Res 39, e35 (2011). 


\section{FIGURES}

\begin{tabular}{|c|c|c|c|}
\hline Mice & Genotype & Sex & Fertility \\
\hline \multirow{2}{*}{ WT (Wild Type) } & \multirow{2}{*}{ Ctcf $^{+/+}$Boris $^{+/+}$} & q & YES \\
\hline & & $\hat{0}^{2}$ & YES \\
\hline \multirow{2}{*}{$\begin{array}{c}\text { CTCF HET } \\
\text { (CTCF Heterozygote) }\end{array}$} & \multirow{2}{*}{ Ctcffl-Boris $^{+/+}$} & 오 & YES \\
\hline & & o & YES \\
\hline \multirow{2}{*}{$\begin{array}{c}\text { BORIS KO (BORIS } \\
\text { Knockout) }\end{array}$} & \multirow{2}{*}{ Ctcf $^{+/+}$Boris $^{-/-}$} & 오 & YES \\
\hline & & 0 & YES(Subfertility) \\
\hline \multirow{2}{*}{ CM ( Compound Mutant) } & \multirow{2}{*}{ Ctcf $^{+-}$Boris $^{-/}$} & q & YES \\
\hline & & $0^{\pi}$ & NO \\
\hline
\end{tabular}

Table1. Summary of in vivo phenotypes associated with different levels of CTCF and BORIS expression. For fertility analysis 3-month-old animals were mated with either the same genotype or with WT. For example, WT and CM males were mated with WT or CM females and vice versa. No offspring were recovered with $\mathrm{CM}$ males. Normal sexual behavior for all genotypes was confirmed by the presence of vaginal plugs in all mated females. 


\section{Figure 1}
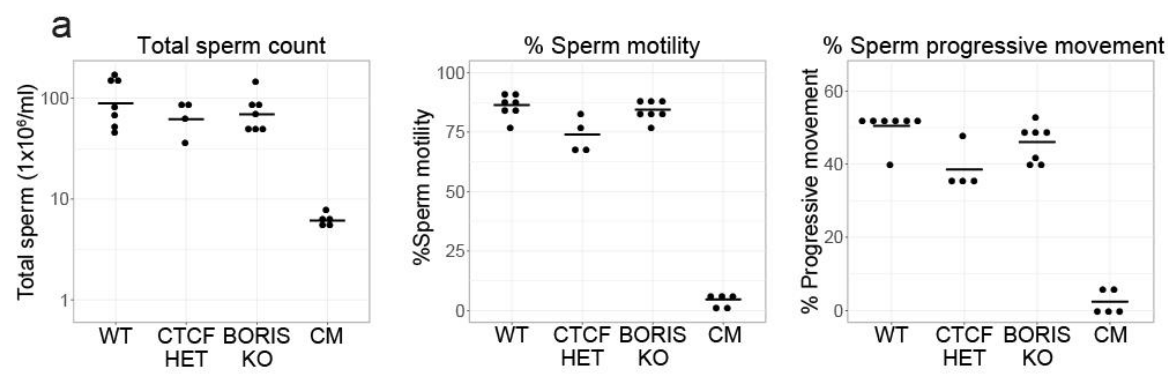

b
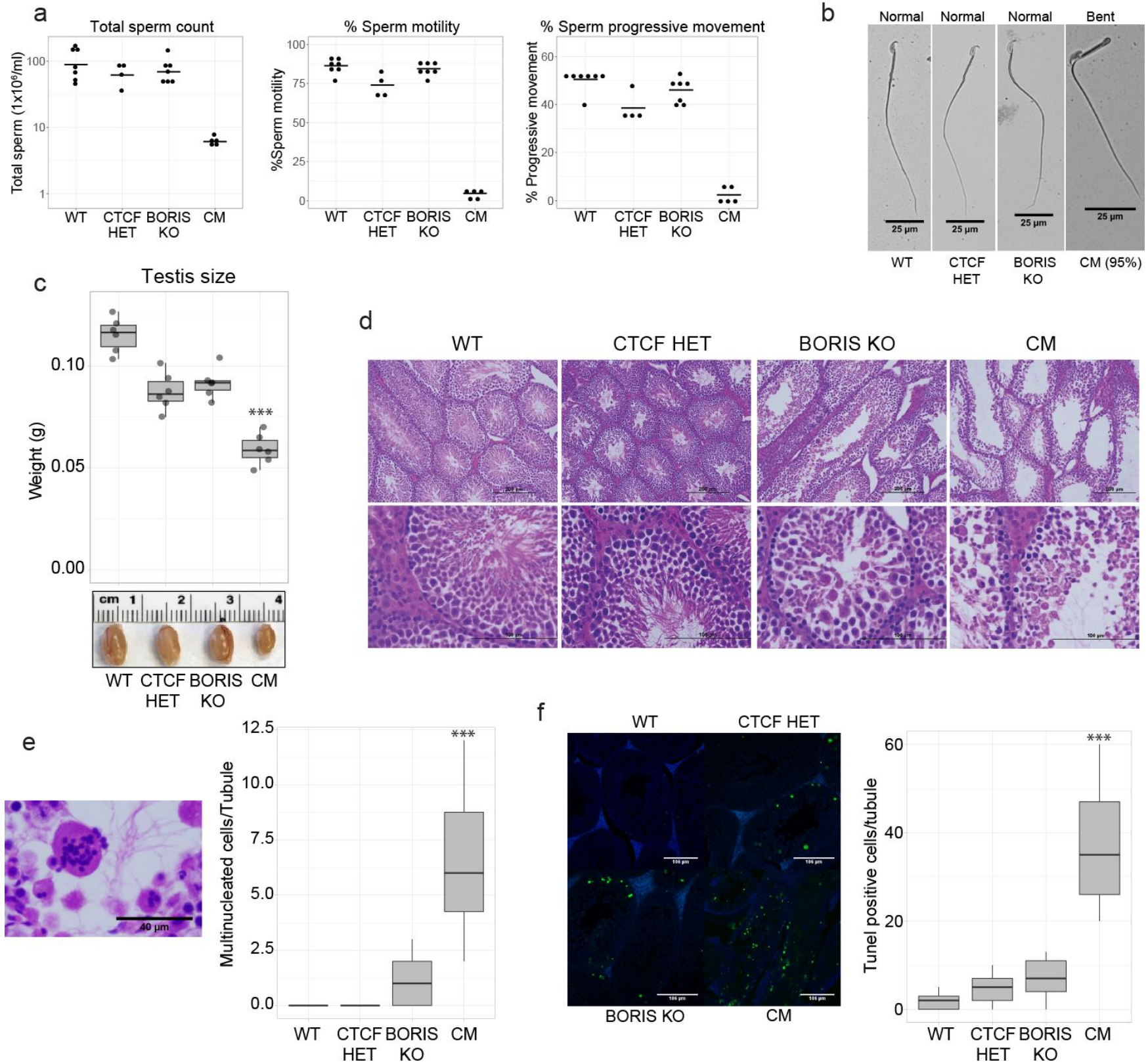

WT CTCFBORIS CM HET KO

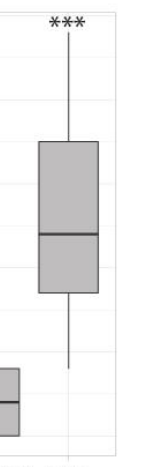


Figure 1. CM mice are infertile with spermatozoa abnormalities and spermatogenic defects. (a). Sperm analysis in the four types of mice (WT; $n=7$, CTCF HET; $n=4$, BORIS KO; $n=7$, and $\mathrm{CM} ; \mathrm{n}=5$ biologically independent animals). From left to right, counts of spermatozoa, $\%$ of motility, and progressive movement. The mean is indicated as a horizontal line. (b). Visualization by light microscopy of spermatozoa from WT, CTCF HET, BORIS KO, and CM mice. The image demonstrates the spermatozoa defects in sterile CM mice with tails bent $180^{\circ}$ through the cytoplasmic droplet. Seven animals from each type of mice were analyzed with similar results. Scale Bars: $25 \mu \mathrm{m}$ (c). Upper panel: Box plot representing the testis weight (grams) in the four types of mice ${ }^{* * *} p$-value $=2.825 \mathrm{e}-07$, Two-sided unpaired t-test equal variance, compared to WT, $n=6)$. Lower panel: representative picture of testis obtained from four types of mice. (d). Hematoxylin and eosin staining of testis prepared from WT, CTCF HET, BORIS KO, and CM 3month-old animals. Seven animals from each type of mice were analyzed with similar results. Scale Bars: $200 \mu \mathrm{m}$ (upper panels), $100 \mu \mathrm{m}$ (lower panels). (e). Left panel: Histological H\&E staining of multinucleated cells present in CM seminiferous tubules. Scale Bars: $40 \mu \mathrm{m}$ Right panel: Box plot representing the quantification of multinucleated cells in the four types of mice ${ }^{* * *} \mathrm{p}$-value $=1.14 \mathrm{e}-12$, two-sided unpaired Mann-Whitney's test, compared to WT, $\mathrm{n}=30,10$ tubules in 3 animals). (f). Left panel: Representative examples of TUNEL staining in the testis from WT, CTCF HET, BORIS KO, and CM mice. Right panel: Box plot representing the quantification of TUNEL positive cells from the four types of mice ( ${ }^{* * *} p$-value $=3.39 \mathrm{e}-31$, twosided unpaired Mann-Whitney's test, compared to WT, $n=90,30$ tubules in 3 animals). Scale Bars: $106 \mu \mathrm{m}$. In the Box plots, the lower and upper hinges correspond to the first and third quartiles, the middle line indicates the median. The upper whisker extends from the hinge to the largest value no further than 1.5 times of the inter-quartile range (IQR). The lower whisker extends from the hinge to the smallest value at most 1.5 times of the IQR. 
Figure 2

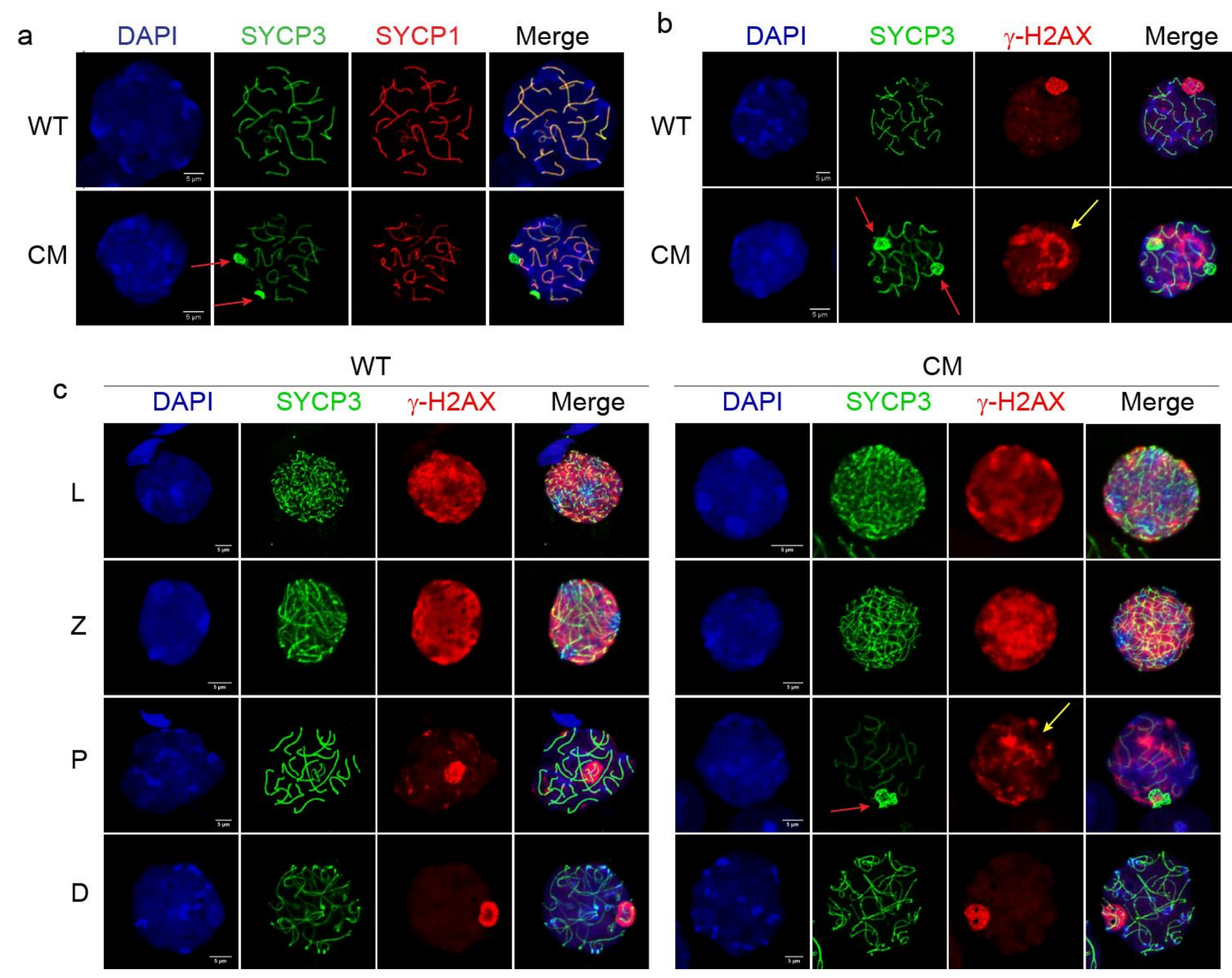


Figure 2. CM mice show defects in progression through meiosis. Spermatocytes prepared from WT and CM mouse testes were fixed and analyzed by immunofluorescence microscopy. (a) Analysis of the synaptonemal complex in WT and CM testes. Spermatocyte spread preparations were stained with anti-SYCP3 (green) and SYCP1 (red) antibodies for visualization of the lateral and central synaptonemal complex components, respectively. The red arrows indicate abnormal SYCP3 staining. (B-C) Spermatocyte spread preparations were stained with anti-SYCP3 (green) and H2AX (red) antibodies. The red arrows show the abnormal SYCP3 staining of pachytene CM spermatocytes. The extension of H2AX staining beyond the sex vesicle in CM spermatocytes is shown by yellow arrows. Three animals from each type of mice were analyzed with similar results. 
Figure 3

a $\log ($ Sum expression in the cluster +1$)$ 8
6 Fraction of cells expressing 4 each gene in each cluster 2000 $0 \quad 00.10 .20 .30 .40 .5$



b
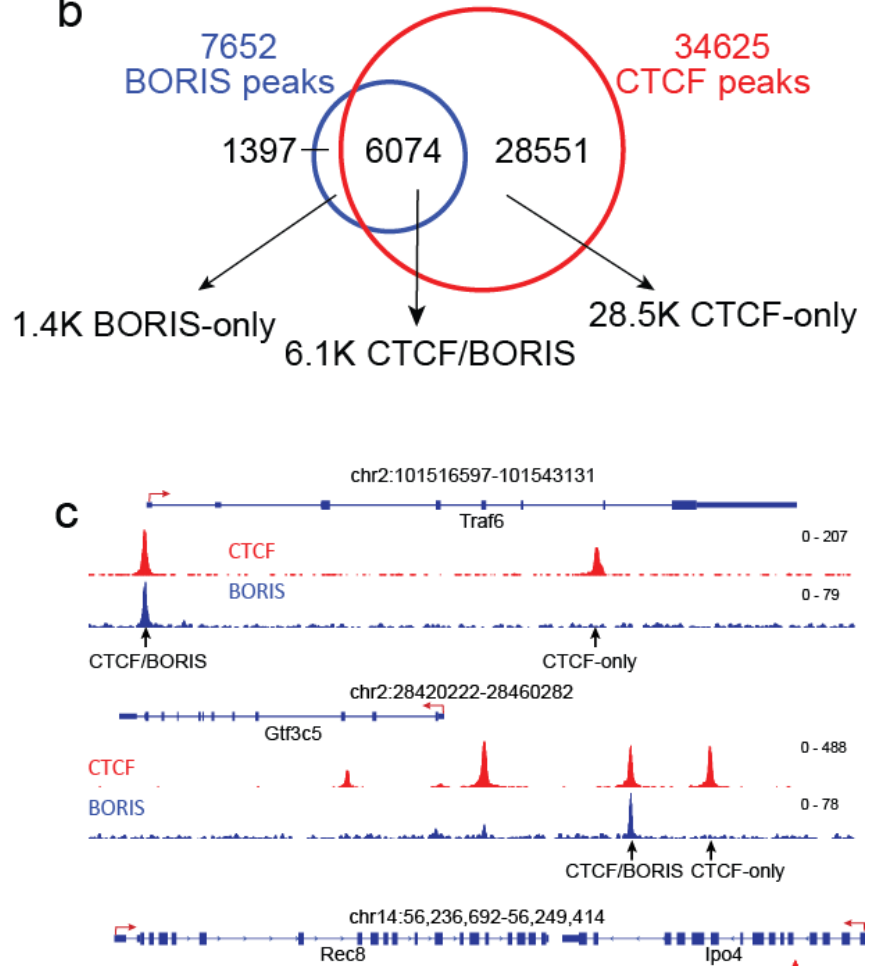

CTCF

BORIS



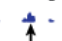

CTCF-only

Active Enhancers

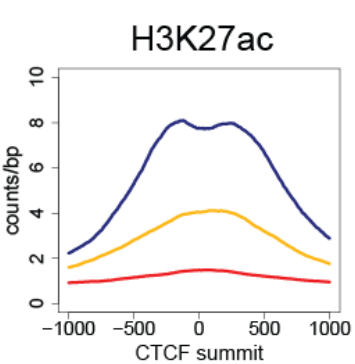

CTCF-only CTCF/BORIS BORIS-only 
Figure 3. CTCF and BORIS cooperate at active promoters and enhancers in male germ cells. (a) Single-cell RNA-seq analysis of Ctcf and Boris expression at the major stages of mouse spermatogenesis, SG: Spermatogonia, SPC: Spermatocytes, RS: Round Spermatids. (b) Venn Diagram depicting the overlap of CTCF (red) and BORIS (blue) occupancy in mouse male germ cells. Based on overlapping of CTCF and BORIS occupancy, 3 clusters were generated: BORISonly, CTCF/BORIS and CTCF-only. (c) Gene tracks of the distribution of CTCF (red) and BORIS (blue) bound regions in mouse male germ cells. (d) Barplot of the genomic distribution of CTCF and BORIS occupancy at promoters + 5'UTR (3kb up and downstream of TSS), gene bodies, and intergenic regions. (e). Average tag density of ChIP-seq data for RNA PollI (RNAPII), H3K4me3 and H3K27ac mapped in mouse testis (ENCODE data) across BORIS-only (blue), CTCF-only (red) and CTCF/BORIS (yellow) bound regions mapped in mouse testis. 


\section{Figure 4}

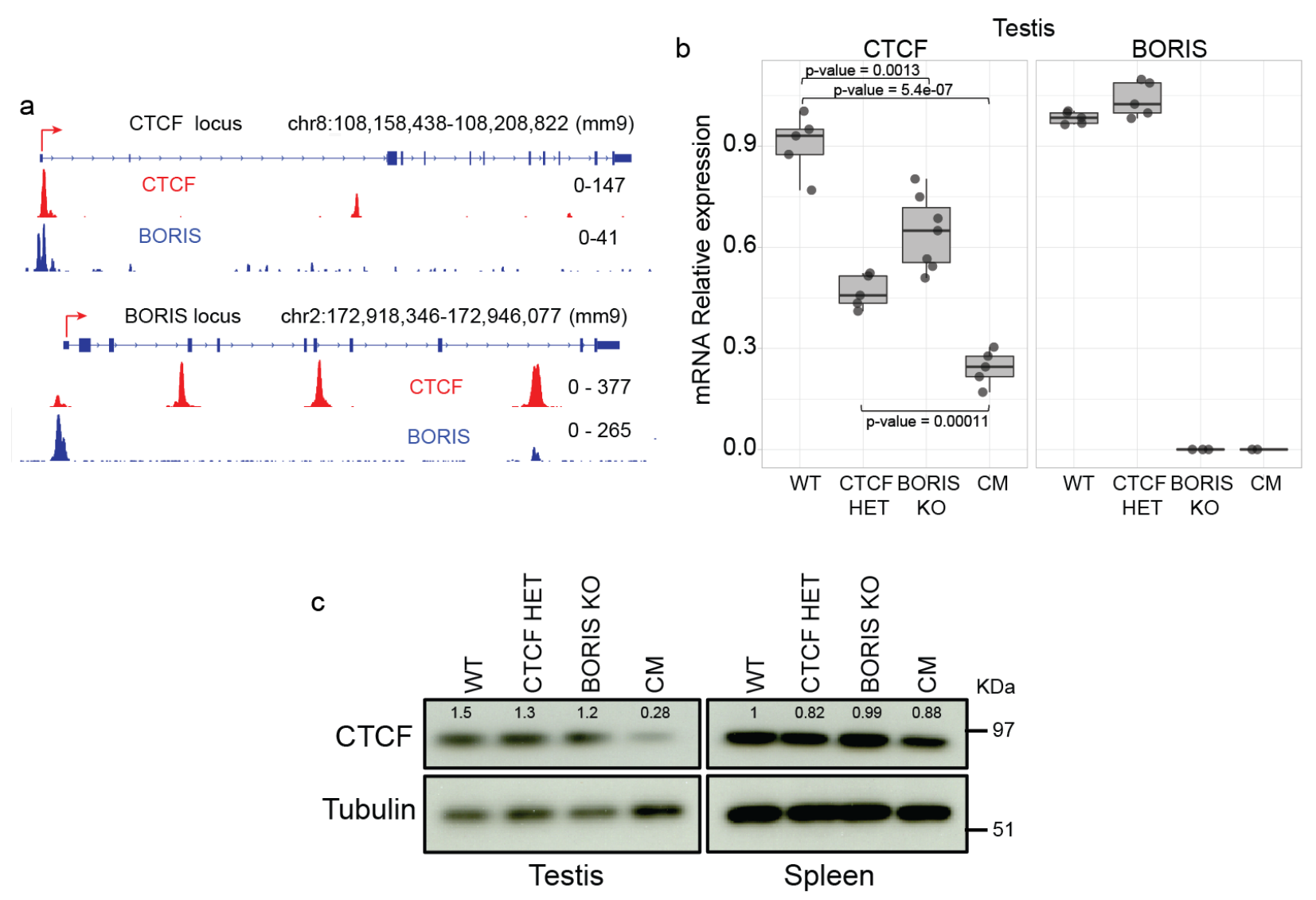


Figure 4. BORIS positively regulates Ctcf gene expression in testis. (a) Genome Browser view of CTCF (red) and BORIS (blue) occupancy at the promoter regions of the Ctcf and Boris genes in WT testes. (b) Expression of Ctcf and Boris analyzed by Real Time PCR in testis of the four mouse genotypes. All data were normalized to Gapdh. Two-sided unpaired t-test was performed, the $p$-values are indicated in the figure (WT, CTCF HET and CM; $n=5$, BORIS KO; $\mathrm{n}=7$ ). The lower and upper hinges correspond to the first and third quartiles, the middle line indicates the median. The upper whisker extends from the hinge to the largest value no further than 1.5 times of the inter-quartile range (IQR). The lower whisker extends from the hinge to the smallest value at most 1.5 times of the IQR. (c) Western blot analysis of CTCF protein levels in testis and spleen from the four types of mice. Tubulin was used as a loading control. Two independent experiments were repeated with similar results. Densitometry values were normalized to tubulin. 
Figure 5

a

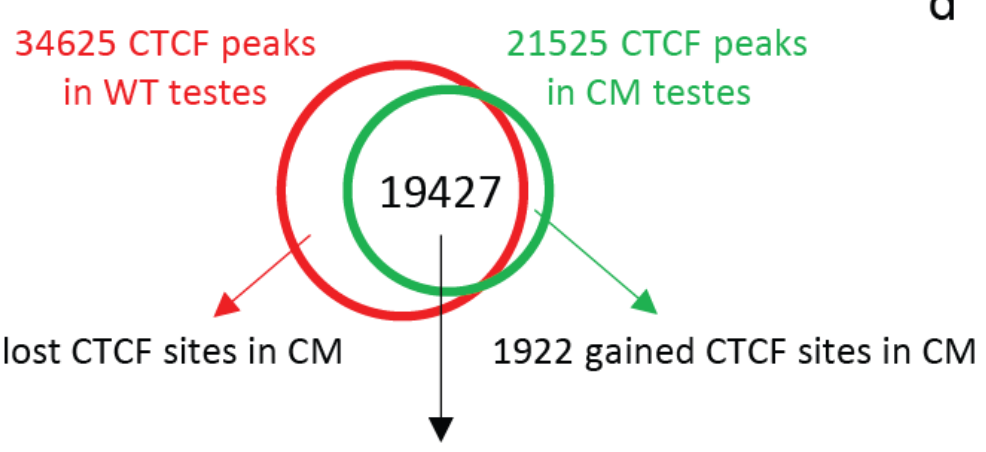

CTCF occupancy at 19427 CTCF sites

b



C

CTCF occupancy at CTCF/BORIS and CTCF-only binding regions

CTCF/BORIS binding regions

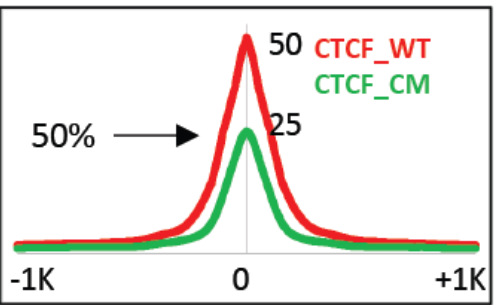

$+1 \mathrm{~K}$
CTCF only-binding regions

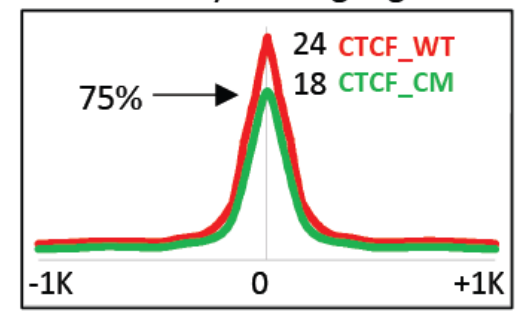

d

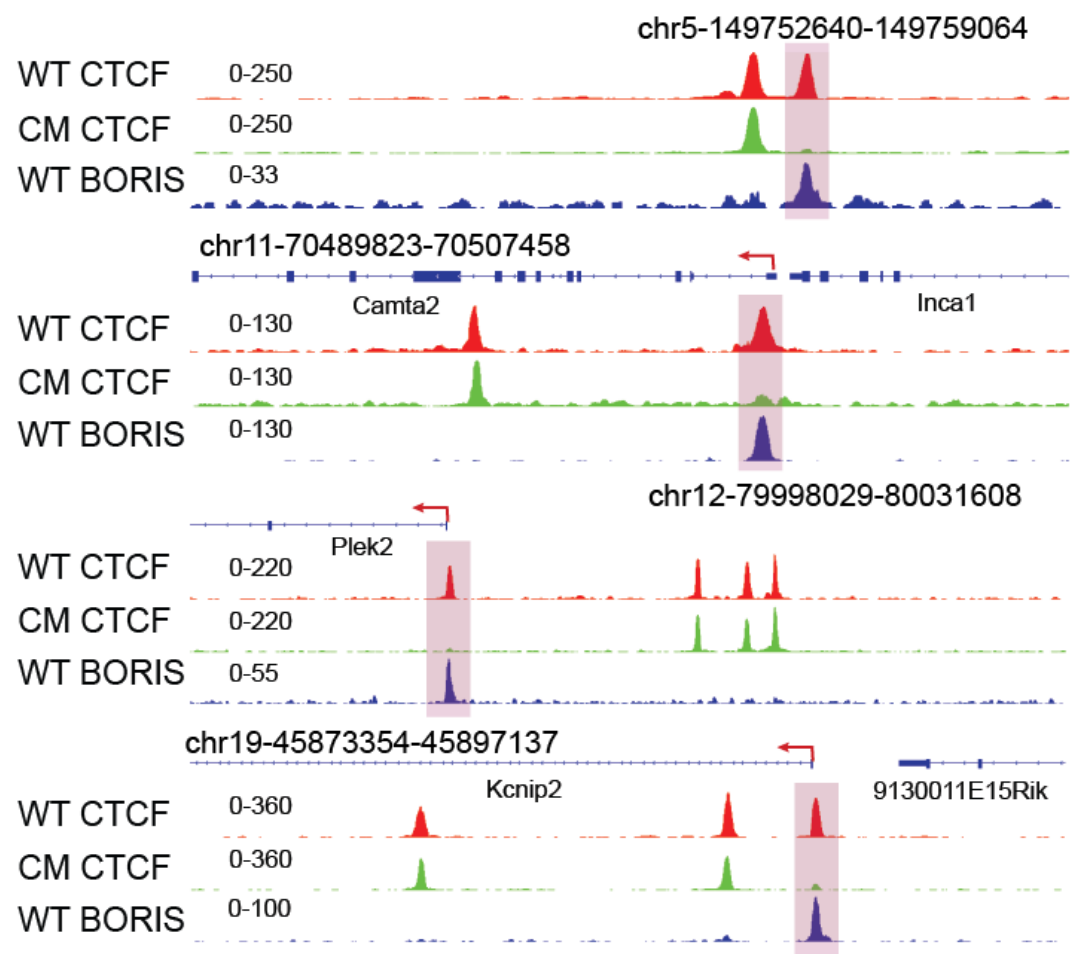

CTCF occupancy in WT and CM testes at

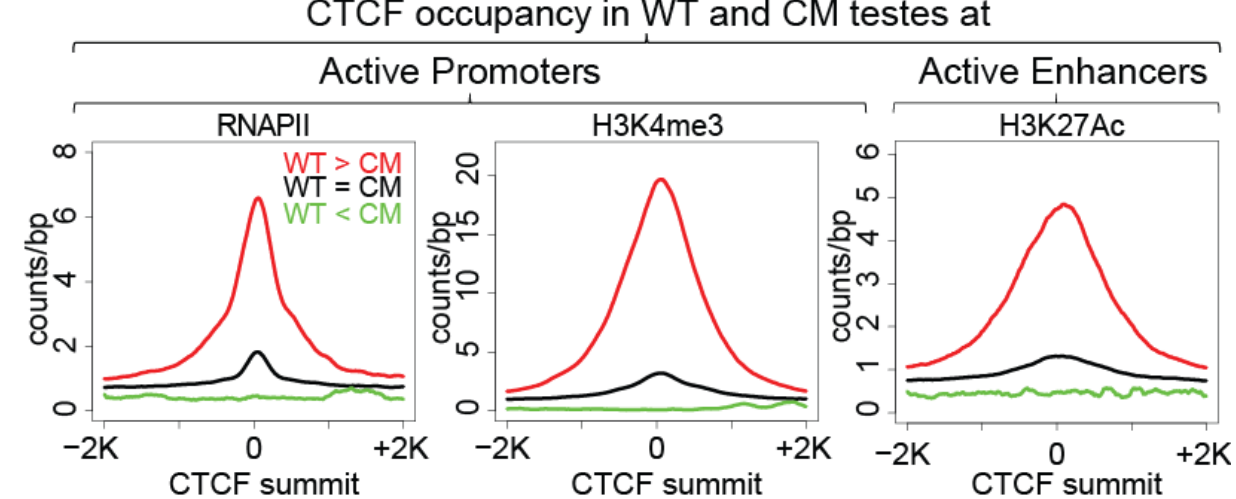


Figure 5. The loss of CTCF binding in CM testes occurs preferentially at CTCF/BORIS heterodimeric sites. (a) Venn diagram shows an overlapping of CTCF ChIP-seq peaks mapped in WT (red) and CM (green) testes. (b) Average tag density of CTCF occupancy in WT and CM testes at 19427 CTCF binding sites. The black arrow shows connection between (a) and (b). (c) Average tag density of CTCF occupancy mapped by ChIP-seq in WT (red) or CM (green) male germ cells across CTCF/BORIS (left panel) and CTCF-only (right panel) bound regions mapped in WT mouse testis. (d) Gene tracks for specific locations on mouse chromosomes 5, 12, 11 and 19 showing the distribution of CTCF (red) and BORIS (blue) bound regions in WT, and CTCF bound regions in CM (green) mouse male germ cells. Highlighted rectangles indicate the loss of CTCF occupancy in CM male germs cells (preferentially at CTCF/BORIS bound regions mapped in WT testes). (e) Differentially bound CTCF regions are associated with active chromatin marks in WT germ cells. Average tag density of RNA PollI (RNAPII), H3K4me3 and H3K27ac mapped by ChIP-seq in mouse testis (ENCODE data) across regions differentially bound by CTCF $(\mathrm{WT}>\mathrm{CM}, \mathrm{WT}=\mathrm{CM}$, and $\mathrm{WT}<\mathrm{CM}$, loss/decrease of CTCF binding in CM compared to WT, no differences in binding, and preferentially bound in $\mathrm{CM}$, respectively). 


\section{Figure 6}
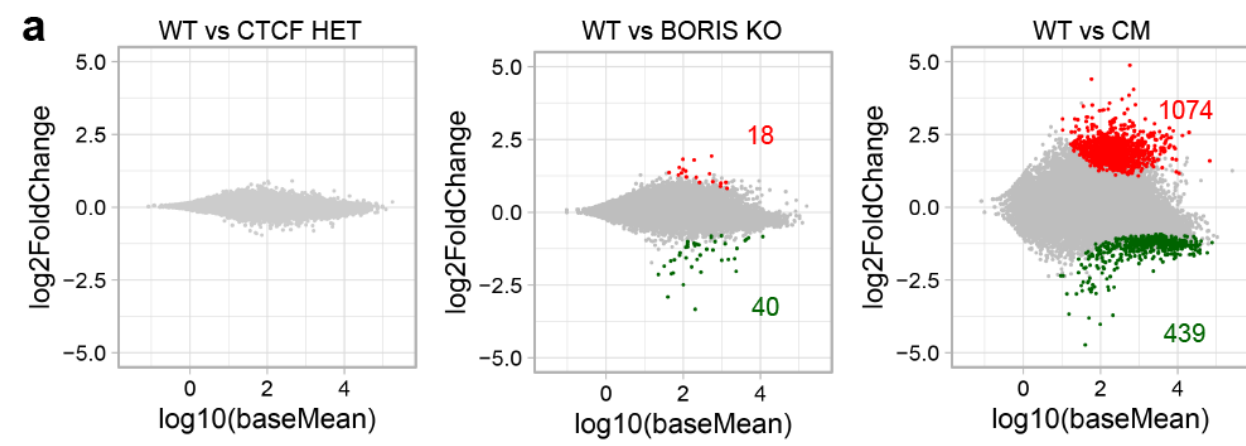

b

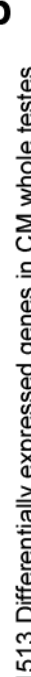

in
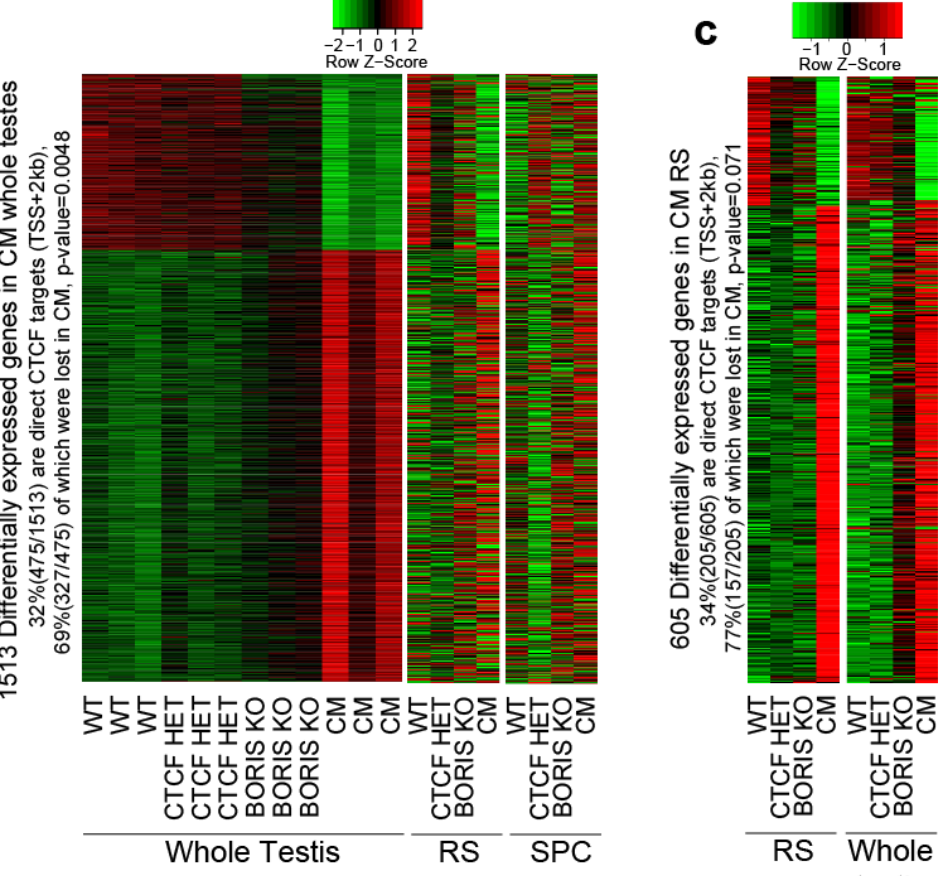

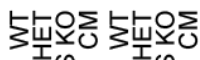

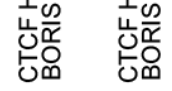

RS Whole

testis



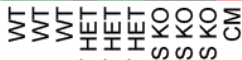

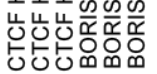

Whole testis

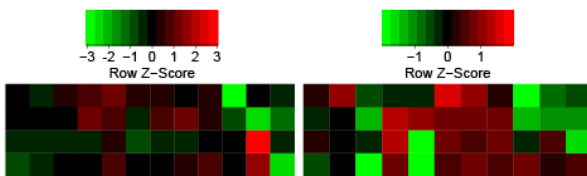

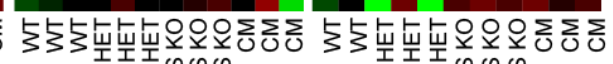

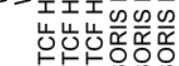

Round spermatids

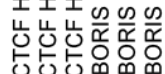

Spermatocytes

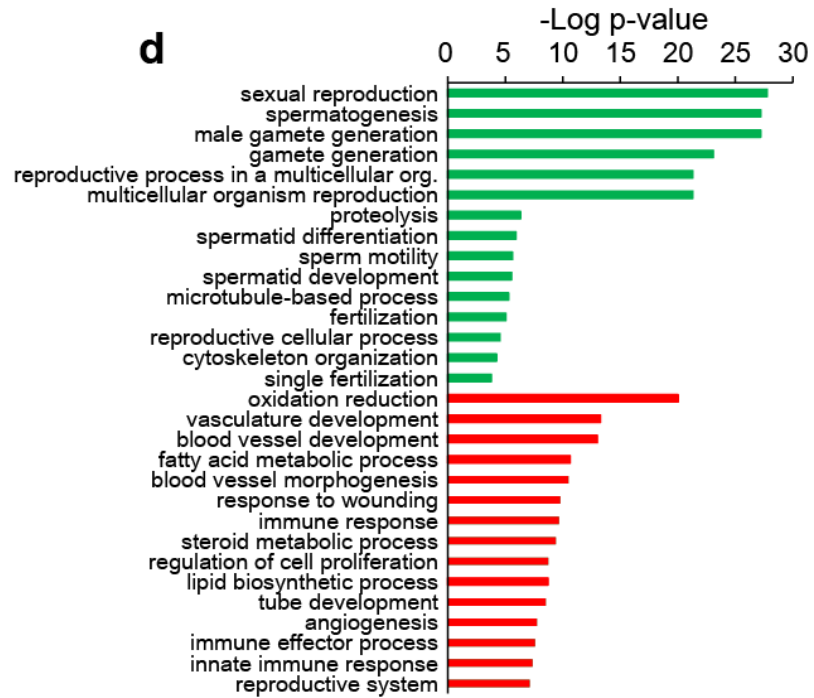

e

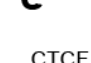

CTCF WT CTCF CM
BORIS WT RNA-Seq WT RNA-Seq
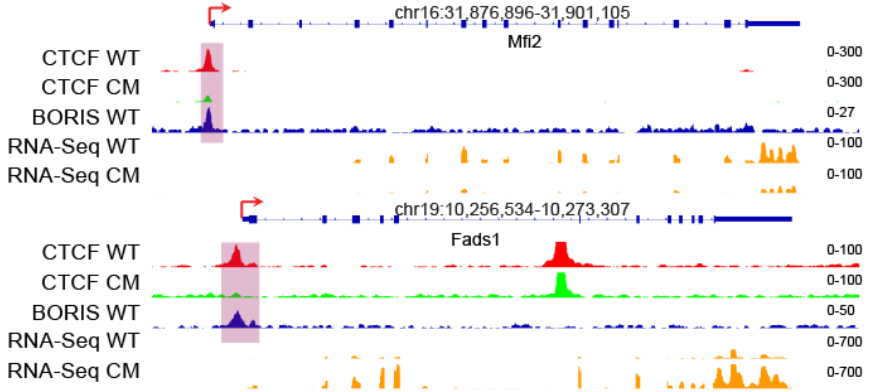
RNA-Seq CM

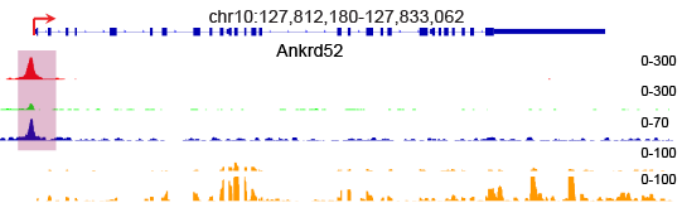


Figure 6. Synergistic effect of CTCF and BORIS deletion on spermatogenesis-specific gene expression. (a) MA plots of RNA-seq in whole testis for CTCF HET, BORIS KO, and CM mice. The $y$-axis represents the log2 of fold change compared to WT; the $x$-axis represents $\log 10$ of mean expression. The red and green dots indicate the up- and down-regulated genes respectively. The gray dots indicate genes without significant changes. The number of differentially expressed genes (DEGs) are highlighted in red (Fold change $>2$ and FDR< 0.001 ). (b) Heat map of DEGs in CM whole testis compared to round spermatids (RS) and Spermatocytes (SPC) in the four types of mice, 3 replicates for each type of mice are represented for whole testis and average of the replicates for RS and SPC. (c) Heat map of DEGs in CM RS compared to whole testis, average of the replicates is shown. (d) Gene Ontology analysis of downregulated (upper panel, green color) and upregulated (lower panel, red color) genes in CM testis compared to WT. The analysis was performed in DAVID Bioinformatics Resources 6.8 (https://david.ncifcrf.gov), Fisher's exact p-values from DAVID software are shown. (e) Genomic tracks displaying CTCF (in WT (red) and CM (green) testes) and BORIS (WT in blue) occupancy in comparison with RNA-seq data (yellow) in WT and CM germ cells across Prss50 (Tsp50), Mfi2, Fads2, and Ankrd52. Red arrows indicate the TSS. (f) Heat map showing the change of expression of the same genes in whole testis, RS and SPC. 


\section{Figure 7}

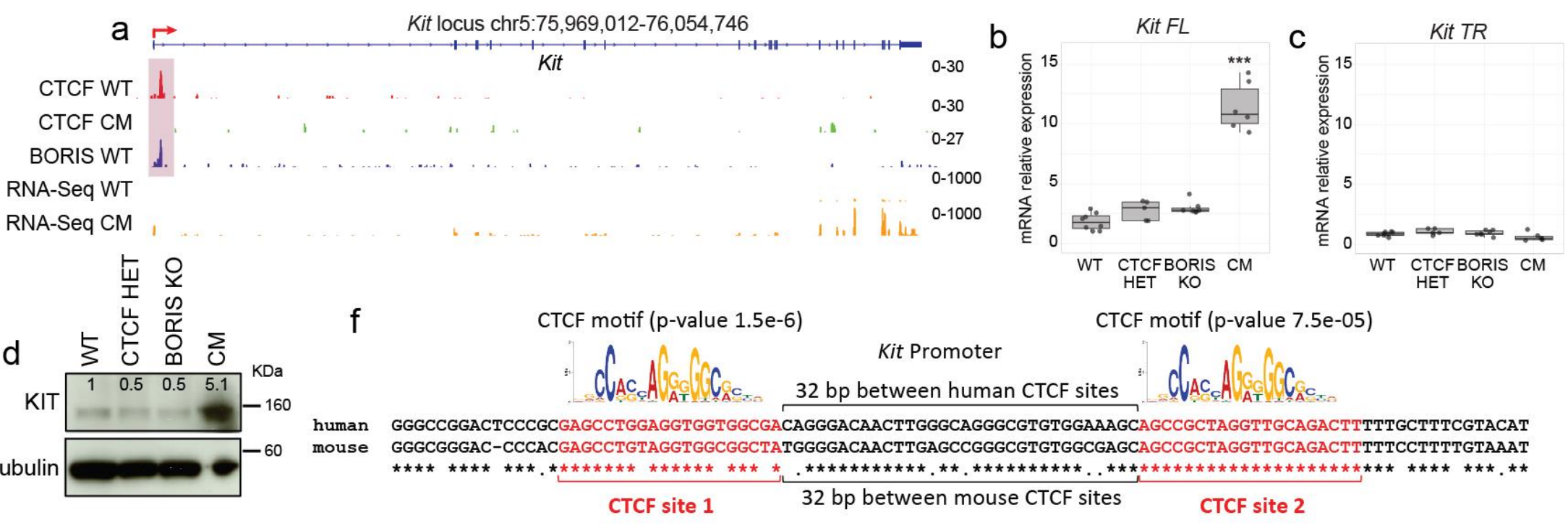

g Human KIT locus chr4:55,519,603-55,535,702

e
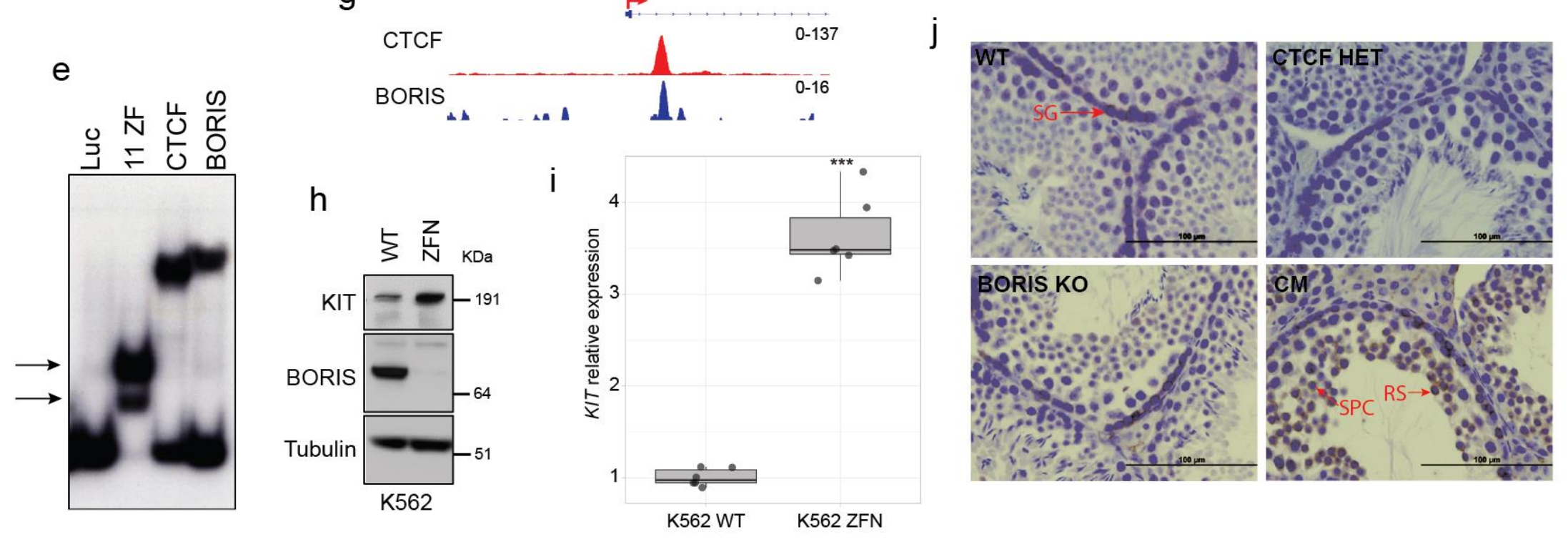
Figure 7. Depletion of BORIS and CTCF expression is associated with toxic Kit persistence. (a) Genomic views of CTCF ChIP-seq in WT (red) and CM (green) testes, BORIS ChIP-seq in WT (blue), and RNA-seq (yellow) in WT and CM testes across the Kit locus. (b-c). Expression of full-length (FL) and truncated (TR) Kit transcript by qPCR in the four types of mice $\left({ }^{\star \star \star} p\right.$-value $=$ 2.93e-08 for Kit-FL, p-value=0.1 for Kit-TR, two-sided unpaired t-test, compared to WT, WT; $n=8$, CTCF HET; $n=4$, BORIS KO; $n=7, C M ; n=6$ ). (d). Western blot of KIT in the four types of testes lysates. Tubulin used as a loading control. Densitometry values were normalized to tubulin and WT $(n=2)$. (e) EMSA of in vitro translated 11ZF domain of CTCF, full-length CTCF and BORIS proteins with DNA fragment comprising CTCF/BORIS bound region at the Kit locus. Luciferase protein (LUC) was used as a negative control. The double-shift of 11ZF domain is indicated by black arrows. The experiment was repeated 3 times. (f). Alignment of human and mouse CTCF/BORIS bound region for the Kit locus. The two 20bp-CTCF motifs were mapped by Motif Alignment and Search Tool. (g) ChIP-seq of CTCF (red) and BORIS (blue) in K562 cells. (h). Western blot of KIT and BORIS in wtK562 and K562 treated with BORIS-specific Zinc Finger Nuclease (ZFN) ( $\mathrm{n}=2)$. (i). qPCR analysis of KIT expression in wtK562 and K652 treated with ZFN. KIT expression is normalized to GAPDH $\left({ }^{* * *} \mathrm{p}\right.$-value $=4.07 \mathrm{e}-08$, two-sided unpaired t-test, compared to WT, n=6) (j). Immunohistochemical detection of KIT in four types of mouse testes. KIT is detected in spermatocytes (SPC) and round spermatids (RS) of CM testis but not in WT; (SG: Spermatogonia). The entire experiment was performed three times. Bars: $100 \mu \mathrm{m}$. In the Box plots, the lower and upper hinges correspond to the first and third quartiles, the middle line indicates the median. The upper whisker extends from the hinge to the largest value no further than 1.5 times of the inter-quartile range (IQR). The lower whisker extends from the hinge to the smallest value at most 1.5 times of the IQR. 